\title{
ДИНАМИКА ИНДЕКСА МАССЫ ТЕЛА РОССИЙСКИХ МУЖЧИН И ЖЕНЩИН: ВОЗРАСТ-ПЕРИОД-КОГОРТА
}

\author{
АНДРЕЙ АИСТОВ, ЕКАТЕРИНА АЛЕКСАНДРОВА, ФАРИДА ГАРИПОВА
}

\begin{abstract}
В работе представлены оценки индивидуальной динамики индекса массы тела (ИМТ) российских мужчин и женщин, выполненные на данных Российского мониторинга экономического положения и здоровья населения НИУ-ВШЭ 1994-2018 г2. Панельная структура данных позволила оценить индивидуальную динамику ИМТ без существенного вклада кросс-секционной составляющей в возрастной профиль ИМТ в рамках эконометрической модели возраст-период-когорта. Эмпирические оченки выявили монотонный рост ИМТ мужчин в 2001-2011 г2. В работе показано, что ИМТ женщин на протяжении жизни растет до 60 лет, мужчин - примерно до 40 лет. Выявлено также, что существует тенденция роста ИМТ в молодых поколениях мужчин. У женщин подобный рост сменяется спадом, начавшимся с поколения женщин 1950 г. рождения. Полученные в работе оценки профилей ИМТ мужчин и женщин в трех измерениях (возраст-период-когорта) могут служить иелям совершенствования политики в области охраны здоровья населения.
\end{abstract}

Ключевые слова: избыточная масса тела, здоровье населения, Россия, панельные данные, РМЭЗ НИУ ВШЭ.

\section{ВВЕДЕНИЕ}

Избыточная масса тела зачастую рассматривается как один из важных факторов заболеваемости и смертности. Так, например, существуют доказательства связи отдельных заболеваний (сердечно-сосудистые заболевания, диабет, болезни легких и некоторые злокачественные новообразования) с избыточной массой тела и ожирением (Rodriguez et al. 2007; Bhaskaran et al. 2014; Arnold et al. 2016; Afshin et al. 2017; Lambert et al. 2017; Wahl et al. 2017; Gu et al. 2019; Kawachi et al. 2019). Ряд авторов обнаруживают увеличение риска смерти человека при отклонении массы его тела от оптимальной величины (Mock et al. 2002; Yi et al. 2015; Di Angelantonio et al. 2016; Cheng et al. 2016; Twig et al. 2016; Afshin et al. 2017; Yu et al. 2017). В работах (Farhat, Iannotti, Simons-Morton 2010) выявлена менее очевидная взаимосвязь: подростки с избыточной массой тела и ожирением более склонны к рискованному образу жизни.

\footnotetext{
АНДРЕЙ ВАЛЕНТИНОВИч АИСТОВ (aaistov@hse.ru), НАЦИОНАЛЬНЫЙ ИССЛЕДОВАТЕЛЬСКИЙ УНИВЕРСИТЕТ «ВЫСШАЯ ШКОЛА ЭКОНОМИКИ», РОсСия.

ЕКАТЕРИНА АЛЕКСАНДРОВНА АЛЕКСАНДРОВА (ea.aleksandrova@hse.r), НАЦИОНАЛЬНЫЙ ИССЛЕДОВАТЕЛЬСКИЙ УНИВЕРСИТЕТ «ВЫСШАЯ ШКОЛА ЭКОНОМИКИ», РОССИЯ.

ФАРИДА ГАБДУЛХАЕВНА ГАРИПОВА (FGaripova@hse.r), НАЦИОНАЛЬНЫЙ ИССЛЕДОВАТЕЛЬСКИЙ УНИВЕРСИТЕТ «ВЫСШАЯ ШКОЛА ЭКОНОМИКИ», РОССИЯ.

ИССЛЕДОВАНИЕ ВЫПОЛНЕНО ЗА СЧЕТ ГРАНТА РОССИЙСКОГО НАУЧНОГО ФОНДА (ПРОЕКТ №20-18-003О7 «ЗДОРОВЬЕ НАЦИИ: ЭКОНОМИЧЕСКИЙ ПОДХОД К ОЦЕНКЕ ЗДОРОВЬЯ И СВЯЗАННЫХ С НИМ НЕРАВЕНСТВА И КАЧЕСТВА ЖИЗНИ НАСЕЛЕНИЯ»).
}

СТАТЬЯ ПОСТУПИЛА В РЕДАКЦИЮ В ЯНВАРЕ 2021 Г. 
Имеются также исследования, посвященные оценкам связи избыточной массы тела человека с его индивидуальными социально-экономическими характеристиками. Согласно некоторым из них избыточная масса тела не всегда является признаком высокого благосостояния (Zagorsky 2005), более того, есть свидетельства, что ожирение снижает вероятность занятости и размер зарплат мужчин (Колосницына, Куликова 2018). Возможными причинами отрицательной связи массы тела и благосостояния могут быть абсентеизм (job/employee absenteeism - привычное, частое отсутствие на рабочем месте) и рост расходов на поддержание здоровья (Bungum et al. 2003).

Перечисленные выше факты говорят об актуальности выявления групп людей, склонных к набору массы тела. Подобного рода исследования могут служить целям совершенствования политики в области охраны здоровья населения.

Следуя критериям, предложенным Всемирной организацией здравоохранения (BO3), классификация людей на группы с избыточной массой тела и ожирением достаточно часто (преимущественно на популяционном уровне) ${ }^{1}$ основывается на вычислении индекса

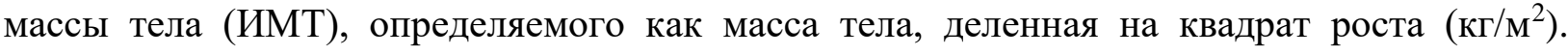
Согласно оценкам ВОЗ взрослый человек имеет избыточную массу тела при выполнении следующего неравенства: $25 \leq$ ИМТ < 30; признаком ожирения является ИМТ $\geq 30$.

Наиболее дискуссионной в настоящее время является задача выявления различий средней величины ИМТ у разных поколений (Hosseini et al. 2017; Wilson, Abbott 2018), поскольку некоторые тенденции могут быть предвестниками массовых отклонений в здоровье, влекущими за собой снижение прогнозируемой продолжительности жизни представителей ряда демографических когорт (Иванова 2014; Badley et al. 2015).

Многие авторы обеспокоены тенденцией роста ИМТ у молодых поколений. Подобные выводы в некоторых работах делаются на основе простого анализа описательных статистик, выполненных на выборках людей разных поколений. Интерпретация подобных результатов имеет определенные сложности. Сравниваемые ИМТ измеряются либо в разные календарные годы, либо в один год, но на разных возрастных когортах. В связи с этим возникает закономерный вопрос о корректном объяснении наблюдаемых эффектов. Является ли рост ИМТ закономерным развитием общества в целом (результатом роста благосостояния, изменения предпочтений, привычек, рациона питания и др.), свойственным всем поколениям, или все-таки мы наблюдаем индивидуальные особенности отдельных поколений, являющиеся, например, результатами различий в их воспитании и укладе жизни, сформированными под влиянием отдельных экзогенных для данных поколений исторических событий?

Целью представленного исследования является идентификация гендерных и когортных особенностей динамики ИМТ, наблюдаемых в России. В рамках предложенного эмпирического исследования мы выделили эффект когорты - эффект года рождения человека. Эмпирическим определением данного эффекта является инвариантное во

\footnotetext{
${ }^{1}$ URL: https://www.who.int/news-room/fact-sheets/detail/obesity-and-overweight 
времени отклонение ИМТ от наблюдаемого среднего уровня, не объясненное в эмпирической модели возрастом человека и годом наблюдения (календарным годом).

Исследование выполнено на данных «Российского мониторинга экономического положения и здоровья населения НИУ-ВШЭ (RLMS-HSE)», проводимого Национальным исследовательским университетом «Высшая школа экономики» и ООО «Демоскоп» при участии Центра народонаселения Университета Северной Каролины в Чапел Хилле и Института социологии РАН ${ }^{2}$.

Эмпирический анализ позволил сформулировать ряд выводов. Эффекты календарного года в 2001-2011 гг. свидетельствуют о монотонном росте ИМТ мужчин при прочих равных условиях (контроле возраста и инвариантных во времени ненаблюдаемых индивидуальных особенностей человека).

Подобных явно выраженных трендов изменений ИМТ женщин на всем протяжении опроса RLMS-HSE не наблюдалось. Оценки изменений ИМТ с возрастом показывают схожую динамику для мужчин и женщин. Различия заключаются лишь в том, что у мужчин выход на плато (прекращение роста ИМТ) наступает раньше, чем у женщин: примерно в 35-40 лет против примерно 55-60 лет. Относительные величины когортных эффектов ИМТ (годов рождения) мужчин и женщин, рожденных в 1915-1925 гг., совпадают: находятся ниже среднего уровня эффекта года рождения на выборках мужчин и женщин соответственно. Также ниже соответствующего среднего уровня находится ИМТ мужчин, родившихся до 1959 г. Женщины 50-х годов рождения имеют ИМТ выше среднего уровня эффектов года рождения. У последующих когорт женщин наблюдается снижение ИМТ с ростом года рождения. Такая тенденция (снижения ИМТ женщин) наблюдается примерно до 1995 г. рождения. ИМТ мужчин в среднем растет практически монотонно с ростом года рождения.

\section{ОБЗОР ЛИТЕРАТУРЫ}

Работ, посвященных динамике ИМТ и сравнению показателей для разных половозрастных групп, достаточно много. В них получены следующие результаты. Наблюдается тенденция роста доли населения с избыточной массой тела и ожирением (Di Cesare et al. 2016; Afshin et al. 2017). При этом отмечаются региональные, гендерные и возрастные различия (Afshin et al. 2017; Di Cesare et al. 2016; Kolarzyk et al. 2012a; 2012b; Wang, Wang 2002). Основные результаты работ по разным странам и различным периодам представлены в таблице П1 Приложения.

Некоторые результаты, полученные по разным странам, схожи. Так, например, средний ИМТ монотонно растет (с возрастом и календарным годом) как у подростков (Kwok et al. 2017; Ogden et al. 2016), так и у взрослого населения (Wilson, Abbott 2018; Jiang et al. 2013; Nooyens et al. 2009; Rosenquist et al. 2014; Caman et al. 2013; Jaacks et al. 2013; Gordon-Larsen, Wang, Popkin 2014; Ng, Corey, Young 2012). Гендерные различия

\footnotetext{
${ }^{2}$ Сайты обследования RLMS-HSE: URL: http://www.cpc.unc.edu/projects/rlms и http://www.hse.ru/rlms
} 
свидетельствуют, что у женщин (преимущественно старших возрастных групп) избыточная масса тела и ожирение наблюдаются чаще, чем у мужчин (Heo et al. 2018; Drøyvold et al. 2006; Jaacks et al. 2013), в то время как в среднем ИМТ у мужчин растет во времени быстрее, чем у женщин (Caman et al. 2013; Maruyama, Nakamura 2018). Тем не менее есть и несколько противоречивые свидетельства. Так, например, в Иране процент детей с ожирением снижается с возрастом (Hosseini et al. 2017), а ИМТ для молодых когорт в Ирландии ниже, чем у старших поколений (Jiang et al. 2013).

Отдельно следует обратить внимание на то, что оценки ИМТ в работах многих авторов приведены отдельно для мужчин и женщин (Kline, Tobias 2008; Maruyama, Nakamura 2018; Колосницына, Куликова 2018). Необходимость такого разделения объясняется существованием гендерных различий как при выполнении медицинских измерений, так и при использовании самооценок. В исследованиях, сравнивающих самооценки роста и массы тела со значениями, полученными с помощью объективных измерений, отмечается, что в среднем мужчины склонны переоценивать свой рост, в то время как женщины склонны недооценивать массу своего тела, что приводит к недооценке ИМТ. При этом самый высокий уровень недооценки ИМТ наблюдается у женщин старших возрастных групп, в то время как наиболее адекватно свой ИМТ оценивают молодые мужчины (Krul, Daanen, Choi 2010).

Среди возможных причин существования гендерных различий ИМТ можно упомянуть различия в предпочтениях потребления продуктов питания. В развитых странах женщины чаще сообщают об употреблении здоровой пищи в отличие от мужчин, в то же время они могут быть в большей степени склонны к потреблению продуктов с высоким содержанием сахара (Wansink, Cheney, Chan 2003; Wardle et al. 2004). Исследования, проведенные в США и Европе, также подтверждают, что рацион питания женщин в большей степени, чем мужчин, состоит из диетических продуктов, в то время как мужчины существенно больше женщин употребляют алкоголь (Kant, Graubard, Kumanyika 2007; Castetbon et al. 2009).

Различия протекания физиологических процессов и строения тела мужчин и женщин также приводят к разным значениям ИМТ. Высокий уровень ИМТ у мужчин в молодом и среднем возрастах отражает более высокую по сравнению с женщинами мышечную массу. При этом увеличение ИМТ тела у женщин в постменопаузе связывают с увеличением жировой массы (Ley et al. 1992).

Анализ динамики ИМТ (особенно при когортном сравнении) имеет несколько «сложностей». В частности, одним из наиболее дискуссионных вопросов является адекватность границ ИМТ для определения избыточной массы тела и ожирения. Следует отметить, что сравнение трех альтернативных критериев определения избыточной массы тела и ожирения, предложенных организацией International Obesity Task Force, IOTF (Cole et al. 2000), Всемирной организации здравоохранения, ВО3 (WHO 1995) и авторами Маст, Даллал и Дитс (Must, Dallal, Dietz 1991a; 1991b), свидетельствует об отсутствии значимых различий возрастной динамики долей подростков с избыточной массой тела и ожирением (Wang, Wang 2002). 
Еще одной особенностью изучения динамики ИМТ и сравнений между группами является проблема кросс-секционных данных, которые занижают оценки ИМТ с возрастом, основанные на поперечном анализе, по сравнению с моделями, учитывающими панельный характер данных (Nooyens et al. 2009). Дискуссионным остается и вопрос о квадратичной зависимости ИМТ от возраста (Wilson, Abbott 2018; Jiang et al. 2013; Колосницына, Бердникова 2009).

Следует также отметить, что большинство работ посвящено анализу ИМТ в разрезе «ИМТ-возраст», «ИМТ-когорта» или «ИМТ-год». Не исключено, что часть противоречивых результатов объясняется невключением третей компоненты для одновременного анализа во всех трех направлениях. Также различия могут быть связаны с межстрановыми или национальными особенностями исследуемых групп населения (Cawley 2004).

Исследования по России свидетельствуют о гендерных различиях: ожирение с большей вероятностью наблюдается у женщин, чем у мужчин (Kalichman, Livshits, Kobyliansky 2006; Hauffman, Rizov 2007; Колосницына, Бердникова 2009; Hu et al. 2017). Исторические данные свидетельствуют о росте ИМТ у детей и подростков (Godina 2011). Наблюдается исторический рост ИМТ (Григорьева 2015), причем у женщин это происходит быстрее, чем у мужчин (Колосницына, Куликова 2018). Избыточную массу тела и ожирение имеют 62-69\% взрослого населения (Gurevich et al. 2017; Колосницына, Куликова 2018). Несмотря на схожесть результатов исследований по России с другими странами, использование авторами методологии, одновременно учитывающей три измерения «возраст - год наблюдения - год рождения» для России, нами не найдено.

Приведенные ниже результаты иллюстрируют гендерные и когортные особенности динамики индивидуальных значений ИМТ населения России по трем измерениям: возраст, период, когорта.

\section{ДАННЫЕ}

Как уже было сказано выше, настоящее исследование выполнено на данных RLMS-HSE

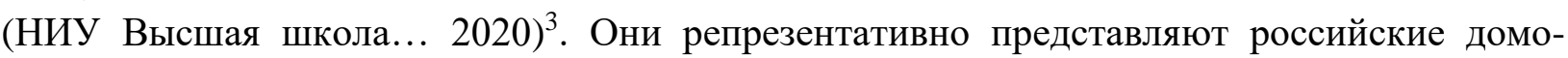
хозяйства, начиная с 1994 г. (в опросе отсутствуют лишь 1997 и 1999 г.). Для достижения целей нашего исследования (см. описываемую ниже эмпирическую модель) принципиальным моментом является использование данных, имеющих значительную панельную составляющую. Этому условию удовлетворяет проект RLMS-HSE.

\footnotetext{
${ }^{3}$ С целью экономии места и ввиду популярности данных RLMS-HSE среди исследователей, мы не будем подробно описывать проект RLMS-HSE, поскольку это было сделано многократно в других работах (обширный список работ размещен на сайте: URL:https://www.hse.ru/rlms/publ). Напомним лишь, что основополагающим мотивом создания проекта RLMS-HSE было желание получать информацию о влиянии рыночных реформ в Российской Федерации на здоровье и благосостояние ее населения.
} 
В рамках настоящего исследования мы использовали все доступные на момент его выполнения волны RLMS-HSE: с 1994 по 2018 г. В выборку были включены участники опроса в возрасте 18-81 год.
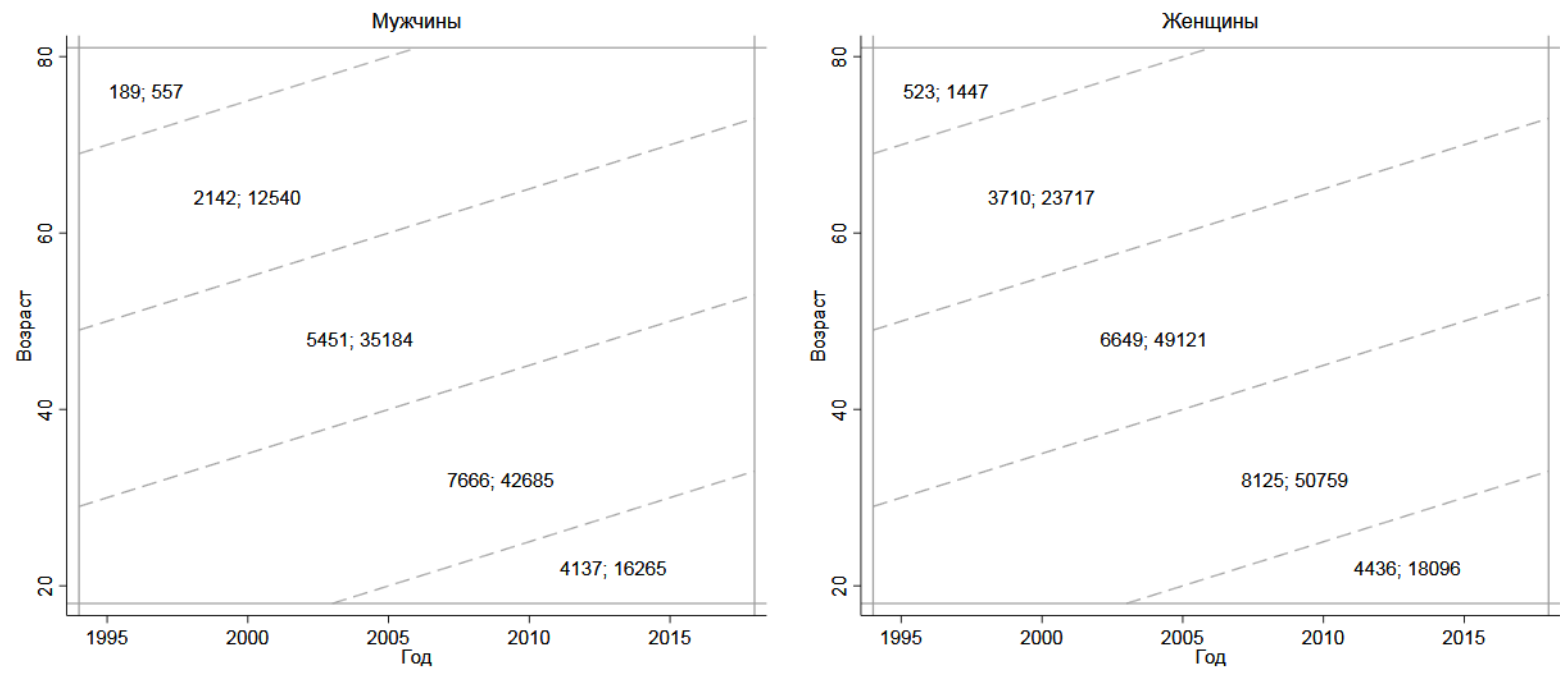

Рисунок 1. Объемы выборок

Источник: Расчеты авторов на основе данных RLMS-HSE (НИУ Высшая школа... 2020).

Примечание: Линии жизни (пунктир) разделяют людей разных годов рождения (поколений): 1925, 1945, 1965, 1985. Числа между линиями жизни показывают количество индивидов, родившихся в соответствующем интервале лет, и (через точку с запятой) число наблюдений.

Анкеты RLMS-HSE содержат вопросы о массе тела и росте респондентов. Используя полученные ответы, мы создали интересующую нас переменную - ИМТ, исключив из выборки лишь 2 сомнительных ответа, в которых мужчина и женщина указали свой рост 70 и 60,5 см соответственно. В результате была получена информация о ИМТ для 98\% мужчин и 97,8\% женщин 18-81 года, участвовавших в опросе RLMS-HSE в 1994-2018 гг. Объемы выборок (число респондентов и количество наблюдений) показаны на рисунке 1 с использованием диаграмм Лексиса (Vandeschrick 2001). Такой вид отображения выбран нами для наглядного представления панельной структуры данных. Чтобы не загромождать рисунок, мы ограничили годы рождения индивидов для подсчета объемов выборок линиями жизни с большими интервалами - 20 лет ${ }^{4}$.

Рисунок 1 дает наглядное представление о структуре данных RLMS-HSE и их высокой панельной составляющей (это видно из сопоставления числа респондентов и количества наблюдений в каждом интервале годов рождения). Панельная структура данных позволяет создавать эмпирические модели, использующие информацию об индивидуальных изменениях ИМТ с возрастом отдельных респондента, не конструируя аналогичные зависимости по представителям разных поколений, как это пришлось бы делать при наличии лишь кросс-секционных наблюдений.

\footnotetext{
${ }^{4}$ В эмпирических оценках нами будут использованы индивидуальные годы рождения без объединения в когорты индивидов нескольких лет рождения.
} 


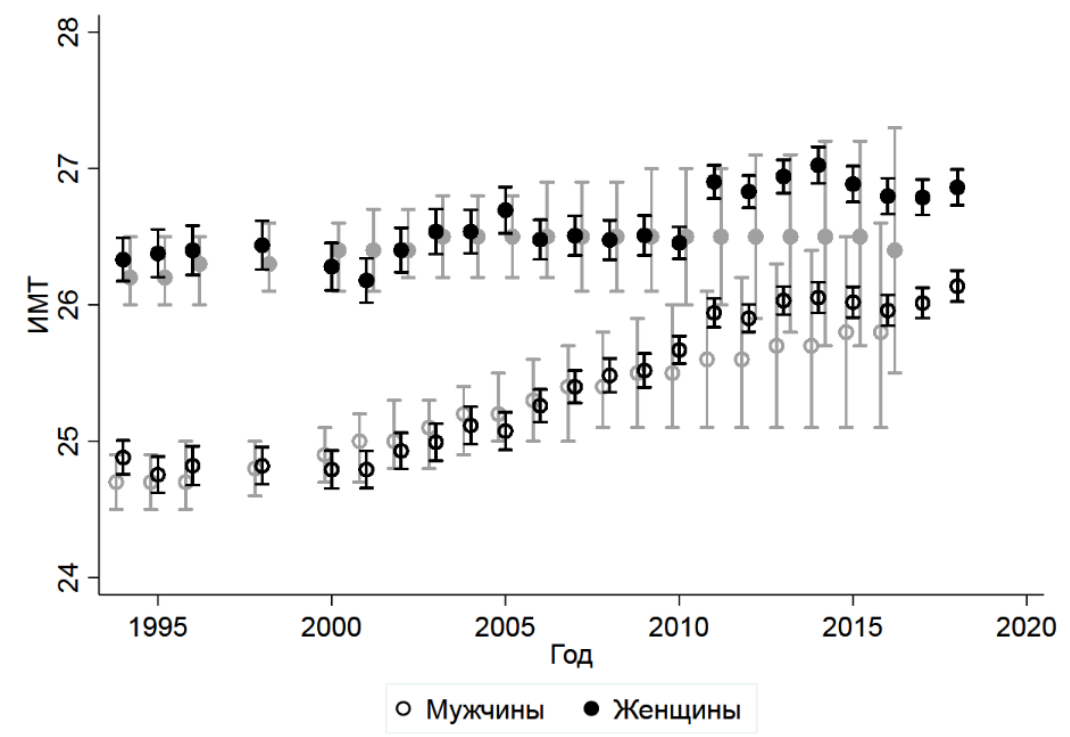

Рисунок 2. Средние значения ИМТ для мужчин и женщин, полученные на выборках RLMS-HSE (черный цвет), и данные ВО3 (серый цвет)

Источник: Расчеты авторов на основе данных RLMS-HSE (НИУ Высшая школа... 2020); данных ВОЗ: URL: https://apps.who.int/gho/data/node.main.A904?lang=en.

Примечание: Вертикальными отрезками показаны 95\%-ные доверительные интервальл.

С целью проверки репрезентативности для России значений ИМТ, полученных на данных RLMS-HSE (самооценках), мы сравнили средние ежегодные значения этого индекса с данными, размещенными на сайте $\mathrm{BO}^{5}$ (инструментальными оценками). На рисунке 2 серым цветом показаны ежегодные средние значения ИМТ, стандартизированные по возрасту (для возрастов 18+), с 95\%-ными доверительными интервалами, размещенные на сайте ВОЗ. Черным цветом обозначены ежегодные оценки значений ИМТ (с соответствующими 95\%-ными доверительными интервалами), полученные авторами на выборке RLMS-HSE. На рисунке 2 видно, что с учетом 95\%-ных доверительных интервалов результаты, полученные из двух источников, совпадают. Незначительные скачки точечных оценок ИМТ, построенных по данным RLMS-HSE (не выходящие за пределы 95\%-ных доверительных интервалов данных ВО3), объясняются изменениями объемов выборки RLMS-HSE (использована несбалансированная панель), происходившими в 2001, 2006, 2010 и 2014 г. (таблица П2 Приложения).

Дальнейший анализ данных с целью выяснения, какие возрастные группы, участвовавшие в опросах RLMS-HSE, дают вклад в оценки, представленные на рисунке 2, удобно выполнить, воспользовавшись диаграммами Лексиса (рисунок 3), на которых с помощью линий уровня (соединяющих равные значения ИМТ) показаны значения ИМТ в зависимости от календарного года и возраста респондентов. При построении рисунка 3 усредненные значения ИМТ были вычислены нами по индивидуальным данным (отдельно для мужчин и женщин) для каждого календарного года и возраста.

\footnotetext{
${ }^{5}$ URL: https://apps.who.int/gho/data/node.main.A904?lang=en
} 

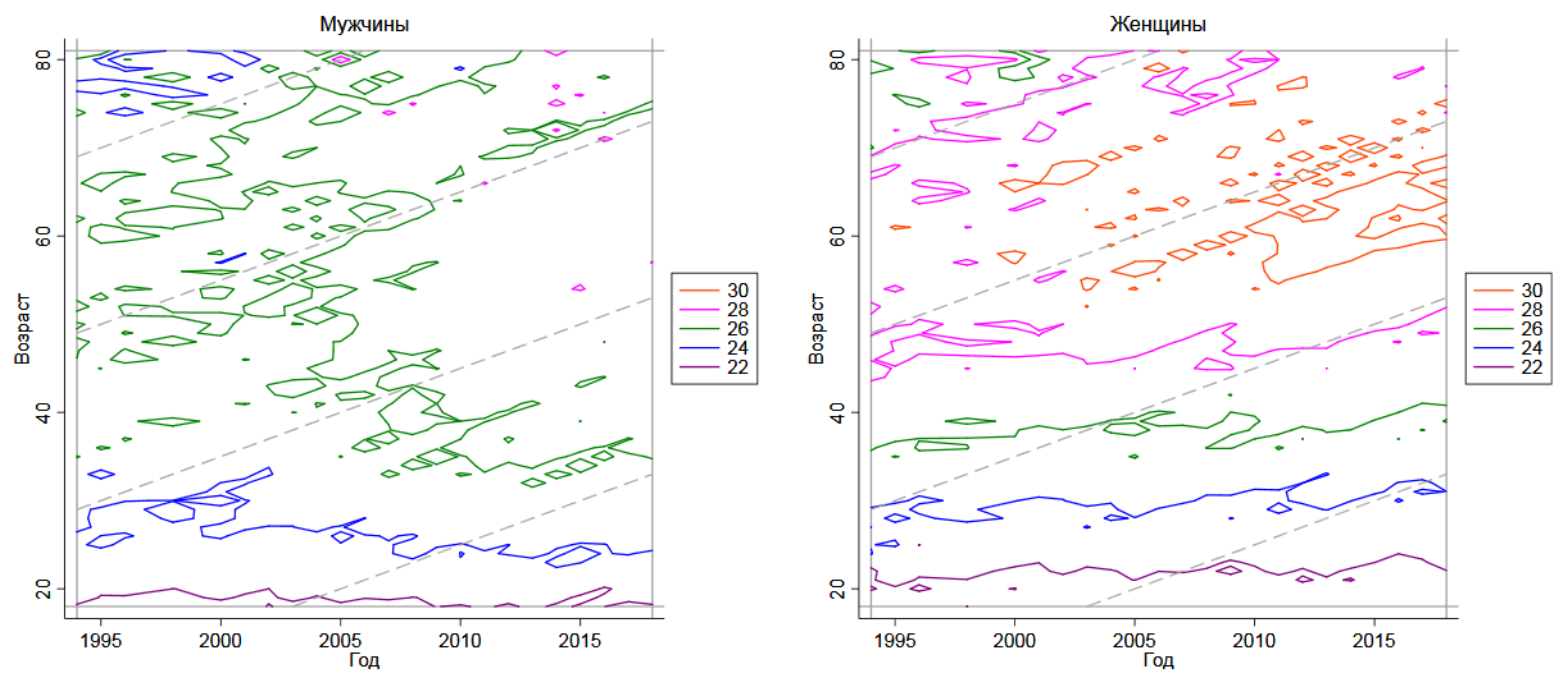

\section{Рисунок 3. Линии уровней ИМТ}

Источник: Расчеты авторов на основе данных RLMS-HSE (НИУ Высшая школа... 2020).

Примечание: Линии уровней ИМТ от 22 до 30 обозначены ияветом. Линии жизни (пунктир) соответствуют рисунку 1.

На рисунке 3 видно, что в некоторых когортных группах мужчин, родившихся в интервале 1966-1984 гг., усредненное значение ИМТ 26 достигается в более раннем возрасте по сравнению с мужчинами 1945-1965 гг. рождения. То же самое можно сказать про достижение уровня ИМТ 28, если сравнивать мужчин 1945-1965 гг. рождения с мужчинами, родившимися в 1925-1944 гг. (рисунок 3).

Аналогичные выводы о достижении высоких значений ИМТ в более раннем возрасте некоторыми представительницами более молодых поколений женщин можно сделать при рассмотрении локализации линий уровня ИМТ 30 в когортах 1925-1965 гг. рождения (правый график на рисунке 3).

Дальнейший анализ данных выполнен с использованием непараметрических оценок. На рисунке 4 показаны зависимости ИМТ от возраста в отдельных когортных группах, различающихся годами рождения. Эти непараметрические оценки получены в эконометрическом пакете Stata методом локального сглаживания с использованием полиномов степени 0, т. е. локальным усреднением (StataCorp 2019a: 293). Весовая функция Епанечникова (StataCorp 2019b: 1210) и ее полуширина (StataCorp 2019b: 1329) выбраны по умолчанию.

На рисунке 4 видно, что ИМТ для некоторых молодых поколений респондентов превышает показатель для старших поколений в соответствующем возрасте. На выборке мужчин это отчетливо проявляется при сравнении зависимостей ИМТ от возраста респондентов, родившихся в 1913-1925, 1926-1945, 1946-1965 и 1966-1985 гг. (рисунок 4). На выборке женщин наивысшее усредненное значение ИМТ в соответствующем возрасте наблюдается у когорты 1946-1965 гг. рождения, вторым по высоте является профиль ИМТ когорты 1926-1945 гг. рождения, остальные профили расположены ниже этих двух (правый график на рисунке 4). 

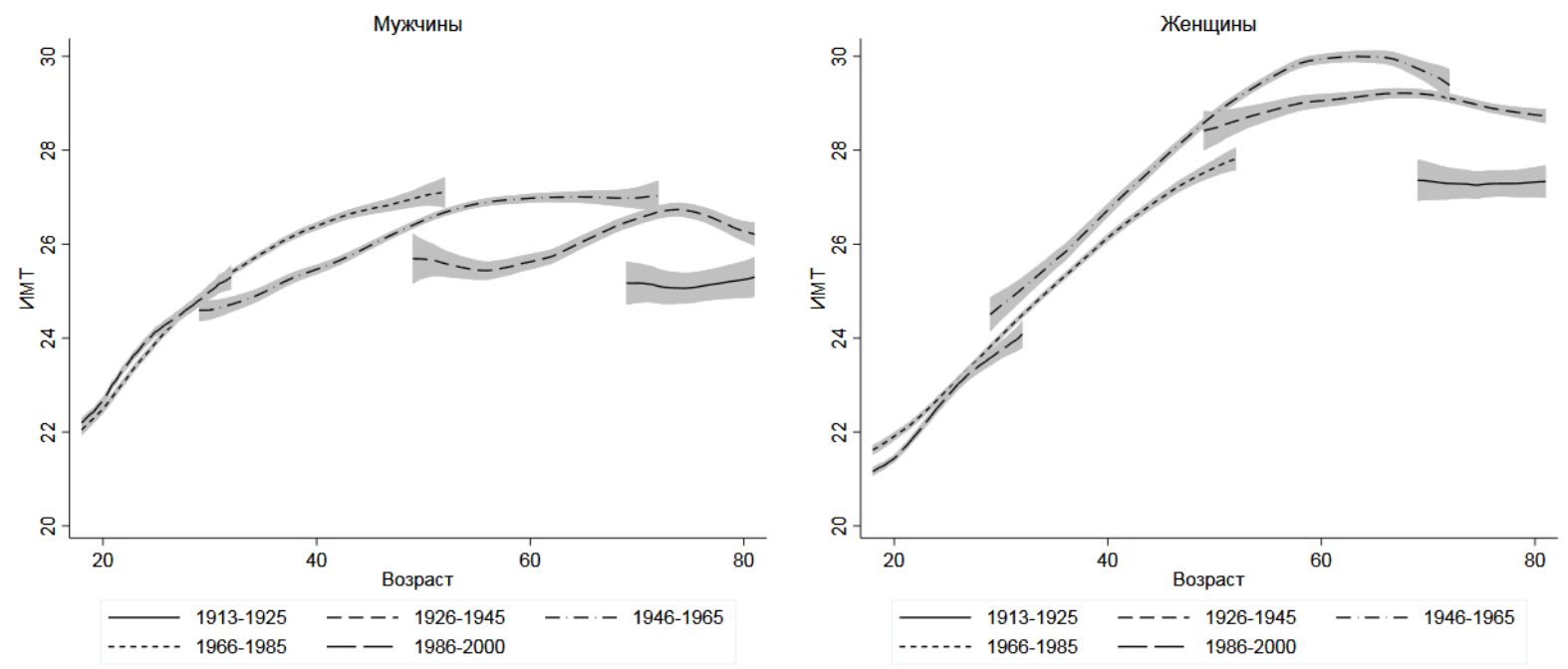

Рисунок 4. Непараметрические оценки зависимости ИМТ от возраста для поколений 1913-1925, 1926-1945, 1946-1965 и 1966-1985 гг. рождения

Источник: Расчеты авторов на основе данных RLMS-HSE (НИУ Высшая школа... 2020).

Примечание: Серым ияветом показаны 95\%-ные доверительные интерваль.

Интерпретируя полученные результаты, следует отметить, что сравниваемые ИМТ измеряются в разные календарные годы (рисунок 3). В связи с этим возникает закономерный вопрос о корректном объяснении наблюдаемых эффектов. Является ли рост ИМТ закономерным развитием общества в целом (результатом роста благосостояния, изменения предпочтений, привычек, рациона питания и др.), свойственным всем поколениям, или все-таки мы наблюдаем индивидуальные особенности отдельных поколений, являющиеся, например, результатами различий в воспитании и укладе жизни, сформированными под влиянием отдельных экзогенных для данных поколений исторических событий? Приведенные ниже эмпирические параметрические оценки выполнены с целью получения ответа на данный вопрос.

\section{ЭМПИРИЧЕСКАЯ МОДЕЛЬ}

Говоря эмпирическим языком, в рамках решаемой нами задачи необходимо разделить эффекты возраста, календарного года и года рождения человека в регрессионных моделях c зависимой переменной ИМТ. Такое разделение осложнено проблемой мультиколлинеарности объясняющих переменных, возникающей при одновременном включении в модель трех линейно связанных регрессоров: возраст человека равен разности календарного года и года рождения.

Обсуждению способов идентификации трех эффектов и получаемым при этом результатам посвящено большое количество работ, в том числе и демографов, рассматривающих так называемую проблему «возраст-период-когорта» (age-periodcohort, APC, problem). 
В настоящее время существует много алгоритмов выполнения оценок параметров APC-моделей. Их краткое описание можно найти в работах (Fu 2016; Аистов 2018; Ethan, Christopher 2019; Su, Lee 2019). Все эти алгоритмы фактически сводятся к наложению ограничений на эмпирическую модель, использующуюся для описания «процесса генерации данных». Например, можно предположить равенство эффектов отдельных возрастов или когорт, если в эмпирической модели используются наборы бинарных переменных для идентификации АРС-компонент (Mason et al. 1973; Yang, Land 2013; Pelzer et al. 2015).

Некоторые авторы, следуя работе (Holford 1983), записывают объясняющие переменные (возраст, период, когорту) в виде ограниченных по абсолютной или относительной величине отклонений от предполагаемых трендов.

Использование смешанных или иерархических моделей также эквивалентно наложению ограничений на модель - выбору, какая из АРС-компонент описывается случайными эффектами (Yang, Land 2006; Dassonneville 2013; Zhang 2015).

Следует заметить, что даже получивший последнее время популярность так называемый intrinsic estimator (Yang, Land 2013; Аистов 2018) использует алгоритм, накладывающий ограничения на коэффициенты при некоторых бинарных переменных, идентифицирующих AРC-эффекты (Pelzer et al. 2015).

Совершенно очевидно, что все известные к настоящему времени алгоритмы дают хорошие результаты, если наложенные на модель ограничения (включая тренды или предполагаемые явные функциональные параметрические зависимости) соответствуют процессу генерации данных (Su, Lee 2019).

Предварительное представление данных (рисунок 4) показывает, что подобрать универсальную функцию с малым количеством параметров, одинаково хорошо описывающую возрастные профили ИМТ респондентов всех поколений, вряд ли возможно. Учитывая этот факт, а также с учетом изменений объема выборки RLMS-HSE, происходивших в отдельные годы ${ }^{6}$, и наличия ненулевого временного тренда ИМТ у женщин (рисунок 2), универсальным простым вариантом записи модели является использование наборов бинарных переменных для идентификации АРС-эффектов. Соответствующая эконометрическая модель, учитывающая панельный характер данных, имеет следующий вид:

$$
y_{i t}=\sum_{j=19}^{81} \delta_{j} A_{i j j}+\mu_{t}+u_{i}+\varepsilon_{i t}
$$

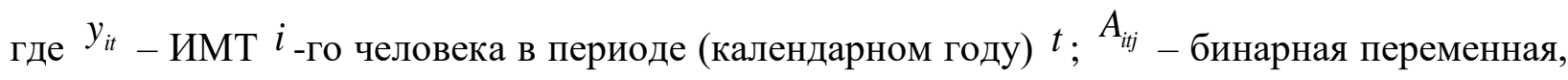
равная 1 , если возраст ${ }^{i}$-го человека в году ${ }^{t}$ равен ${ }^{j}, 0$ - в противном случае; пределы суммирования по индексу $j$ ограничены минимальным и максимальным возрастом индивидов, для которых выполнено настоящее исследование, 18 лет при этом выбрано в

\footnotetext{
${ }^{6}$ Наиболее сильные скачки панельной составляющей (дополнение и уменьшение числа людей, участвующих в опросе) наблюдались в 2006, 2010 и 2014 г. (таблица П2 Приложения). 
качестве базовой категории $\left(\delta_{j}-\right.$ эффекты возраста); $\mu_{t}$ - временные фиксированные эффекты (эффекты календарного года), идентифицируемые с помощью набора соответствующих бинарных переменных (1994 г. при этом был выбран нами в качестве базовой категории); ${ }^{u_{i}}$ - ненаблюдаемые инвариантные во времени (неизменные в 1994-

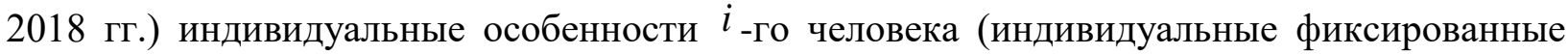
эффекты); $\varepsilon_{i t}$ - случайное слагаемое, изменяющееся как между индивидами, так и во времени; $i=1,2, \ldots, n ; t=1,2, \ldots, T$.

Одной из целей настоящего исследования является оценка фиксированных эффектов $u_{i}$, характерных для людей, родившихся в разные годы. Для этого мы использовали опрос RLMS-HSE, имеющий значительную панельную составляющую (большая доля индивидов участвует в этом опросе ежегодно на протяжении многих лет). Подробный анализ, подтверждающий относительно низкое истощение панельной составляющей выборки RLMS-HSE во времени, можно найти, например, в работе (Kozyreva, Kosolapov, Popkin 2016).

К сожалению, использование панельных данных вызывает проблему коллинеарности регрессоров, идентифицирующих календарный год наблюдения и возраст человека в модели (1). Для устранения коллинеарности упомянутых регрессоров мы исключили 2002 г. из набора бинарных переменных, идентифицирующих временные фиксированные эффекты $\mu_{t}$. Эта калибровка модели эквивалентна предположению о равенстве эффектов периода в 1994 и 2002 г. следующему из результатов, представленных на рисунке 2 .

После наложения указанного выше ограничения на модель параметры $\delta_{j}$ и $\mu_{t}$ модели (1) оцениваются методом наименьших квадратов с использованием внутригруппового преобразования (Wooldridge 2010), исключающего ненаблюдаемые индивидуальные эффекты $u_{i}$.

Когортным эффектом в рамках модели (1) является среднее значение оценок $\hat{u}_{i}$, полученное усреднением по респондентам с одинаковым годом рождения ${ }^{7}$. Оценки $\hat{u}_{i}$ для этого находятся из модели (1) после выполнения оценок $\hat{\delta}_{j}, \hat{\mu}_{t}$.

Эмпирические оценки в рамках настоящего исследования мы выполнили отдельно для мужчин и женщин, следуя аргументам, приведенным в обзоре литературы.

\footnotetext{
7 Эта инвариантная во времени составляющая ИМТ не объяснена в модели (1) ни возрастом человека, ни календарным годом.
} 


\section{Результаты}

Оценки параметров модели (1) приведены в таблице П3 Приложения. Полученные в рамках этой модели результаты (прогнозы значений зависимой переменной при соответствующих значениях периода, возраста и года рождения) представлены на рисунках 5-7.

Эффекты календарного года (рисунок 5) согласуются с данными ВОЗ (рисунок 2) с учетом доверительных интервалов, показанных на рисунке 2 серым цветом. Согласно этим оценкам (рисунок 5) в 2001-2011 гг. наблюдался монотонный рост ИМТ мужчин. Заметим, что в модели (1) выполнена корректировка с учетом эффектов возраста и инвариантных во времени (в 1994-2018 гг.) ненаблюдаемых индивидуальных особенностей респондентов (включающих когортные эффекты). Вспоминая исторические события, заметим, что отмеченный выше рост ИМТ мужчин наблюдался в период стабильного развития российской экономики в 2001-2008 гг. и выхода из кризиса 2008-2009 гг.

Подобных явно выраженных (с учетом доверительных интервалов) трендов изменений ИМТ женщин на всем протяжении опроса RLMS-HSE не наблюдалось (рисунок 5). Следует отметить, что некоторые скачки оценок ИМТ женщин в некоторые годы (рисунок 5) являются результатами изменений выборки RLMS-HSE (таблица П2 Приложения).

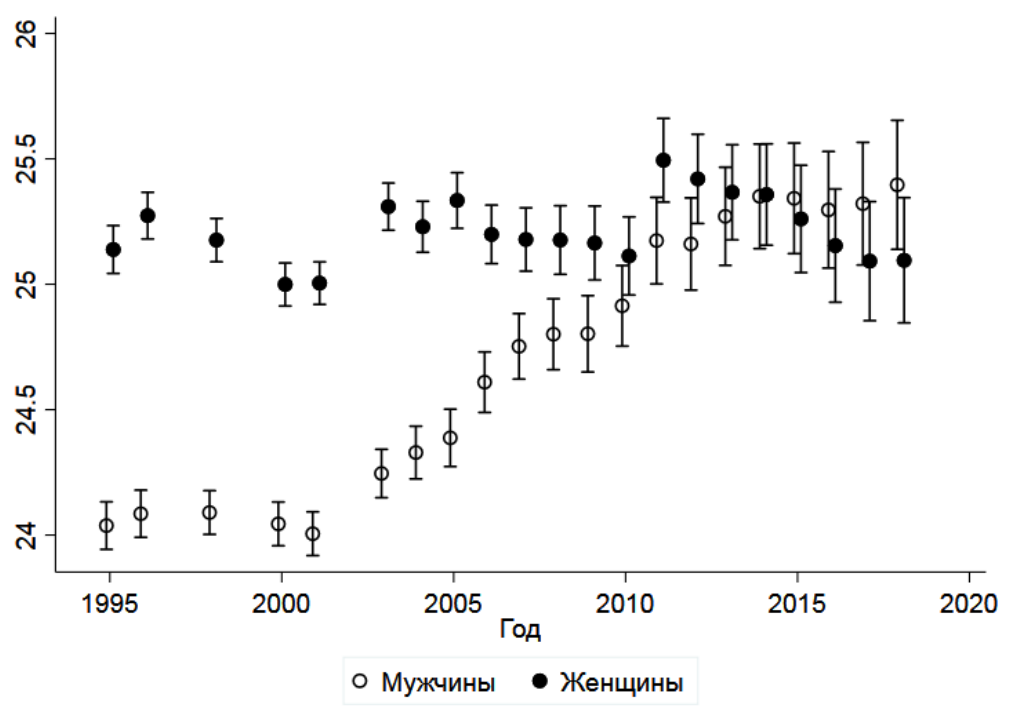

\section{Рисунок 5. Зависимости ИМТ для мужчин и женщин от календарного года}

Источник: Расчеты авторов на основе данных RLMS-HSE (НИУ Высшая школа... 2020).

Примечание: Зависимости получены в рамках модели (1). Вертикальные отрезки - 95\%-ные доверительные интерваль.

Оценки изменений ИМТ с возрастом, выполненные на данных RLMS-HSE (рисунок 6), показывают схожую динамику для мужчин и женщин. Различия заключаются лишь в том, что у мужчин выход на плато (прекращение роста ИМТ) наступает раньше, чем у женщин: примерно в 35-40 лет против примерно 55-60 лет. 


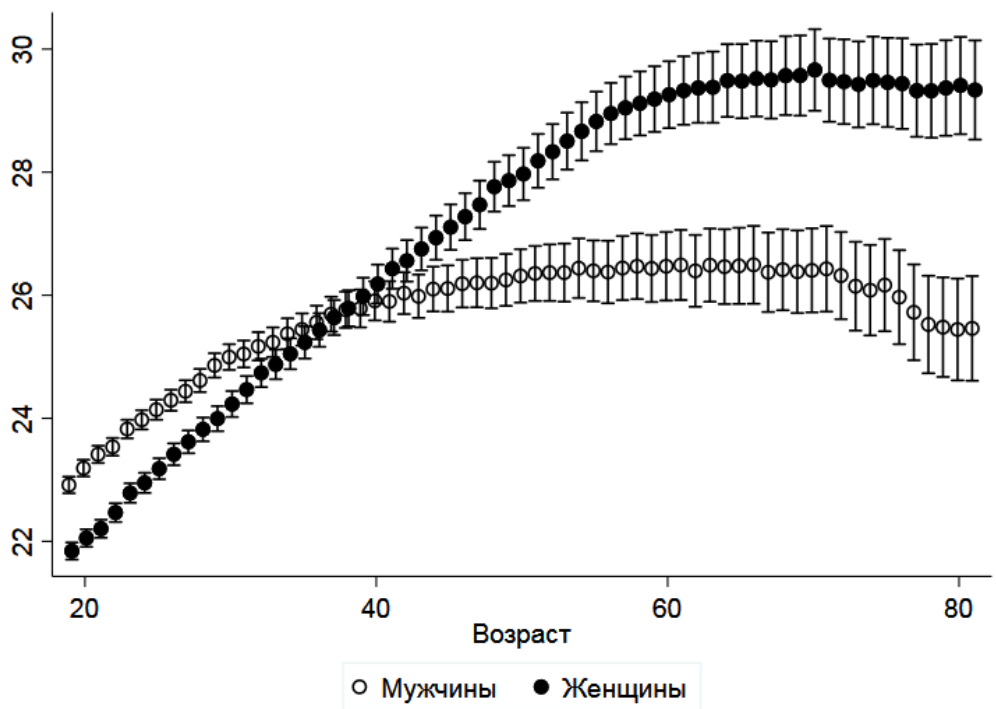

Рисунок 6. Зависимости ИМТ для мужчин и женщин от возраста

Источник: Расчеты авторов на основе данных RLMS-HSE (НИУ Высшая школа... 2020).

Примечание: Зависимости получены в рамках модели (1). Вертикальные отрезки - 95\%-ные доверительные интервальл.

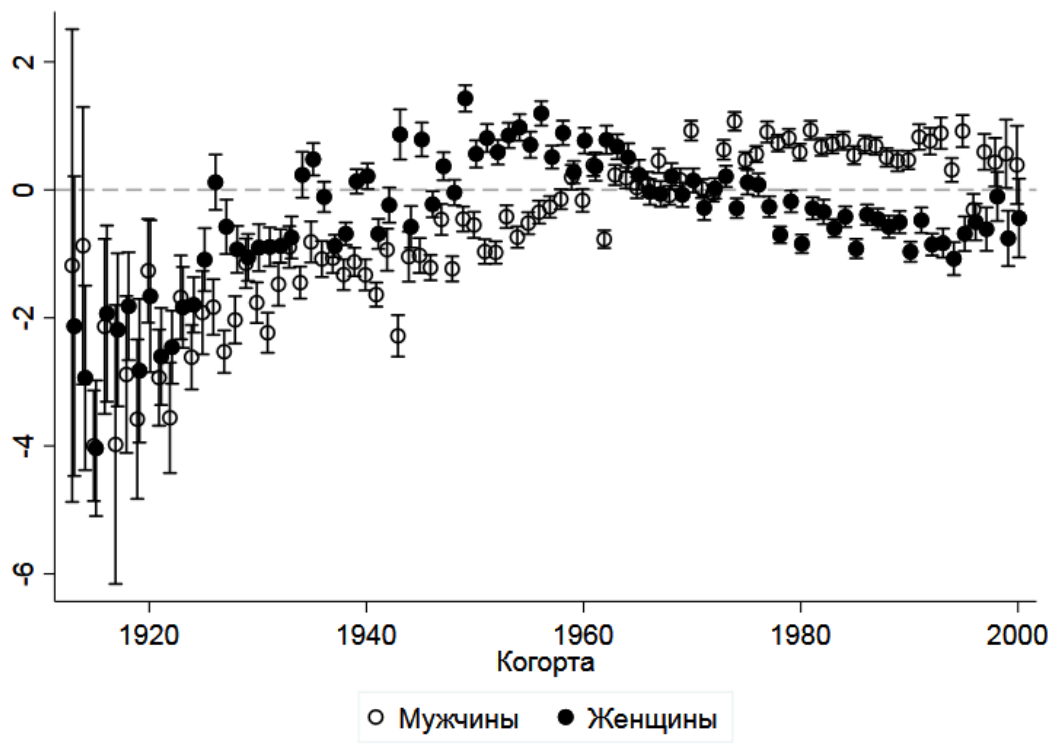

\section{Рисунок 7. Зависимости ИМТ для мужчин и женщин от года рождения (когортные эффекты)}

Источник: Расчеты авторов на основе данных RLMS-HSE (НИУ Высшая школа... 2020).

Примечание: Зависимости получены в рамках модели (1). Вертикальные отрезки - 95\%-ные доверительные интерваль. Отклонения от нуля по вертикальной оси - в единицах ИМТ. Средние значения когортных эффектов равны нулю.

Когортные эффекты (зависимость оценок $\hat{u}_{i}$ от годов рождения участников опроса RLMS-HSE) представлены на рисунке 7. Эти параметры модели включают все 
инвариантные на протяжении используемой нами выборки наблюдений индивидуальные эффекты (неизменные в 1994-2018 гг.), не объясненные изменением возраста человека и года наблюдения.

На рисунке 7 видно, что ИМТ для мужчин и женщин, рожденных в 1915-1925 гг., совпадают (с учетом доверительных интервалов) и лежат ниже среднего уровня, отмеченного пунктирной линией. Также ниже среднего уровня находится ИМТ у мужчин, родившихся до 1959 г. Женщины 50-х годов рождения имеют ИМТ выше среднего. У последующих когорт женщин наблюдается снижение ИМТ по мере увеличения года рождения. Такая тенденция (снижения ИМТ женщин) наблюдается примерно до 1995 г. рождения. ИМТ для мужчин практически на всей выборке RLMS-HSE в среднем почти монотонно растет (за исключением отдельных выбросов). В результате ИМТ для мужчин превышает отмеченный на графике (рисунок 7) нулевой уровень, начиная с когорты 1974 г. рождения.

\section{ОБСУЖДЕНИЕ РЕЗУЛЬТАТОВ}

Анализируя полученные результаты, хочется отметить, что использование самооценок (данных о массе тела и росте) для вычисления ИМТ, судя по литературе и рисунку 2, не приводит к качественным (статистически значимым) изменениям полученных нами результатов по сравнению с ситуацией, если бы мы использовали инструментальные оценки. Возможные смещения значений ИМТ, полученных на основе самооценок, анализировали, например, авторы работы (Kuczmarski, Kuczmarski, Najjar 2001) на выборке из 7772 мужчин и 8801 женщины старше 19 лет (использовались данные NHANES III). Они пришли к выводу, что самооценки роста и массы тела состоятельны для взрослых людей моложе 60 лет. Для старших возрастов самооценки дают немного заниженный результат. Например, для людей старше 70 лет ИМТ, рассчитанный по самооценкам пациентов, был на единицу ниже, чем измеренный клинически.

Авторы работы (Danubio et al. 2008) на выборке молодых людей (студентов двух итальянских университетов), показали, что самооценки ИМТ могут быть занижены примерно на 1,1-1,5 единицы ИМТ (верхняя граница - для женщин) по сравнению с медицинскими измерениями. Заметим, что представленная на рисунке 6 разница оценок ИМТ мужчин и женщин старших возрастов больше 1,5 единиц.

Сравнение самооценок и аппаратно измеренных значений ИМТ было выполнено также в работе (Roth et al. 2013) на выборке 60 женщин репродуктивного возраста. Они обнаружили приемлемую точность самооценок их роста и массы тела. Разница между самооценками и клиническими измерениями была незначительной.

Несмотря на то, что использование самооценок не приводит к статистически значимому смещению оценок ИМТ, в рамках нашего исследования мы будем все-таки говорить об относительных изменениях ИМТ (в зависимости от календарного года, возраста, года рождения). При этом хочется обратить внимание на выявленные нами гендерные различия эффектов возраста, периода и когорты (в последнем случае речь идет о мужчинах и женщинах, родившихся после 1945 г.). В обзоре литературы были 
перечислены некоторые факторы, объясняющие гендерные различия эмпирических оценок на выборках мужчин и женщин. Если говорить о возрастных профилях ИМТ (рисунок 6), то причинами различий могут быть прежде всего особенности протекания физиологических процессов и строения тел мужчин и женщин (Ley et al. 1992). Кроме этого, возможны завышения самооценок роста мужчинами и занижение массы тела женщинами, преимущественно старших возрастов (Krul, Daanen, Choi 2010).

Ряд авторов отмечают, что существуют различия рационов питания мужчин и женщин (Wansink, Cheney, Chan 2003; Wardle et al. 2004; Kant, Graubard, Kumanyika 2007; Castetbon et al. 2009). К сожалению, в рамках проведенного нами исследования не удается дать однозначный ответ, в каком из трех измерений (возраст-период-когорта) предпочтения в выборе продуктов питания играют наибольшую роль в объяснении гендерных различий ИМТ.

Говоря об эффекте периода (рисунок 5), интересно отметить, что данные RLMS-HSE охватывают годы формирования рыночных экономических отношений в России. В связи с этим заметим, что представленные на рисунке 5 тенденции относительного снижения ИМТ женщин по сравнению с мужчинами согласуются с выводами работ (Di Cesare et al. 2016; Maruyama, Nakamura 2018), в которых показано, что в развитых странах ИМТ для женщин в среднем ниже, чем ИМТ для мужчин. Данные RLMS-HSE позволили наблюдать соответствующие наметившиеся тенденции относительного изменения ИМТ в динамике на примере экономики России переходного периода (рисунок 5).

В дополнение к сказанному хочется отметить, что сравнительный анализ ежегодных изменений ИМТ для мужчин и женщин, представленных на рисунке 5, позволяет выдвинуть гипотезы, требующие дальнейшей эмпирической проверки. До 2001 г. - в период активной, стрессовой для многих слоев общества, перестройки экономической жизни - ежегодные тенденции изменений ИМТ для мужчин и женщин, согласно представленным на рисунке 5 точечным оценкам (без учета доверительных интервалов), повторяли друг друга: рост и спад наблюдались одновременно. В периоды относительно спокойного развития экономики, в годы стабильного ежегодного роста ИМТ среди мужчин и выхода их ИМТ на постоянный уровень, наблюдались тенденции (согласно точечным оценкам без учета доверительных интервалов) снижения ИМТ среди женщин. Показанные на рисунке 5 изменения ежегодной сравнительной динамики ИМТ для мужчин и женщин, произошедшие в 2001 г., могут быть темой будущих более тщательных исследований.

Представленные на рисунке 6 результаты отличаются от работ предыдущих авторов (Колосницына, Бердникова 2009; Wilson, Abbott 2018) отсутствием предположения о квадратичной зависимости ИМТ от возраста. Отказ от использования параметрически заданной функциональной формы для возрастного профиля ИМТ в модели (1) позволяет увидеть тенденции (см. точечные оценки для мужчин, показанные на рисунке 6), согласующиеся с так называемым «парадоксом ожирения» (Harrington, Gibson, Cottrell 2009; Lainscak et al. 2012). Возможное объяснение этого парадокса связано со снижением массы тела перед смертью человека в некоторых ситуациях. В частности, авторы работы (Harrington, Gibson, Cottrell 2009) на основе выполненного ими метаанализа пришли к выводу, что вероятность смертельного исхода может быть снижена больными людьми 
благодаря их усилиям, направленным на борьбу с ожирением, но для здоровых людей с избыточной массой тела целенаправленные действия по ее снижению могут привести к обратному эффекту - повышению вероятности смерти. То есть резкое снижение ИМТ в пожилом возрасте может быть предшественником смерти человека. Получение статистически значимого подтверждения этого эффекта может быть темой отдельного исследования.

Многие авторы обеспокоены тенденцией роста ИМТ среди молодых поколений. Подобные выводы в некоторых работах делаются на основе простого анализа описательных статистик, выполненных на выборках людей разных поколений. Использованная нами в рамках настоящей работы простая эмпирическая модель, идентифицирующая эффекты календарного года и возраста, не выявила столь тревожной тенденции на выборке женщин, родившихся с середины 1970-х до середины 1990-х годов (рисунок 7).

Конечно, существует много факторов, объясняющих динамику ИМТ в России. В рамках настоящего исследования мы не использовали методологии выполнения эмпирических оценок, позволяющих выявить соответствующие причинно-следственные связи. Мы можем перечислить лишь основные факторы ${ }^{8}$, которые могут объяснить наблюдаемую динамику ИМТ: изменение структуры занятости (например, снижение доли работников, занимающихся физическим трудом), различие динамики курения мужчин и женщин (по данным RLMS-HSE), изменение диетических предпочтений, продвижение концепции здорового образа жизни и др. При этом хочется подчеркнуть, что воздействие факторов, неизменных у людей на протяжении использованной нами выборки RLMS-HSE 1994-2018 гг., проявляется (согласно смыслу модели с индивидуальными фиксированными эффектами) в когортных эффектах. К таким факторам можно отнести, например, разные уровни образования, доходов, разные вероятности выхода на рынок труда и предпочтения определенных форм занятости, различие склонностей к здоровому образу жизни людей, родившихся в разные годы.

\section{ЗАКЛЮЧЕНИЕ}

В рамках настоящего исследования получены оценки динамики ИМТ с возрастом, годом наблюдения и когортой (годом рождения) человека. Оценки выполнены на данных RLMSHSE с использованием FE-моделей, учитывающих панельный характер данных.

Обзор литературы позволяет сформулировать следующие выводы. Во-первых, усредненные оценки показывают, что в последние десятилетия в большинстве стран мира (за исключением азиатских стран с высокими доходами, европейских и англоязычных стран) средний ИМТ у женщин выше, чем у мужчин. Во-вторых, в большинстве стран наблюдаются тенденции роста доли населения с ожирением. В-третьих, на выборках людей,

\footnotetext{
${ }^{8}$ Авторы благодарны анонимным рецензентам за соответствующие подсказки и возможность цитирования их советов.
} 
принадлежащих разным когортным группам, определяемым годами рождения, наблюдается разная динамика ИМТ во времени.

Описательная статистика использованных нами данных в целом схожа с работами других авторов. В частности, по крайней мере после 1995 г., наблюдается рост ИМТ, при этом у женщин он незначителен, тогда как у мужчин устойчиво растет, начиная с 2001 г. (рисунок 2). С учетом календарного года/года рождения и возраста у женщин ИМТ в среднем выше (см. линии уровня 28 и 30 на рисунке 3); у женщин ИМТ превышает значение 26 в возрасте 40+, а значение 30 в возрасте 50+ (рисунок 3). Значения ИМТ для некоторых молодых поколений респондентов превышает ИМТ для старших поколений в соответствующем возрасте. На выборке мужчин это отчетливо видно при сравнении зависимостей ИМТ от возраста у респондентов, родившихся в 1913-1985 гг. (рисунок 3). На выборке женщин наивысшее значение ИМТ в соответствующем возрасте наблюдается у когорты 1946-1965 гг. рождения (рисунок 3).

Проведенный эконометрический анализ позволил уточнить полученные дескриптивные результаты. В частности, в 2001-2011 гг. наблюдался монотонный рост ИМТ только у мужчин (рисунок 5). Изменение ИМТ с возрастом имеет схожую динамику для мужчин и женщин, но выход на плато (прекращение роста ИМТ) у мужчин наступает в 35-40 лет, а у женщин в - 55-60 лет (рисунок 6). ИМТ у мужчин и женщин 1915-1925 гг. рождения на 2-4 единицы ниже уровня, объясняемого в регрессионной модели возрастом и годом наблюдения (рисунок 7). Подобное отклонение вниз, но меньшее по величине, сохраняется у мужчин до 1959 г. рождения (рисунок 7). У женщин, родившихся в 19501995 гг., наблюдается снижение ИМТ с ростом года рождения - речь идет о когортных эффектах, не объясненных возрастом и годом наблюдения (рисунок 7). У мужчин 1975-1995 гг. рождения когортные эффекты оказались положительными - выше уровня, объясненного возрастом и годом наблюдения.

Основным выводом из проделанной нами работы является утверждение, что полученные для России результаты являются достаточно оптимистичными в отношении молодых поколений женщин. Речь при этом идет о средних значениях ИМТ у женщин, родившихся в 1970-1990 гг. (рисунок 7). Если же говорить об угрозе «глобального ожирения» населения, наличие которой может предполагаться, исходя из высоких оценок ИМТ для молодых поколений, полученных некоторыми авторами (см., например, работу (Di Cesare et al. 2016) и обзор литературы), то начатое нами исследование может быть продолжено. Мы не анализируем распределение ИМТ среди населения (не выделяем доли людей с избыточной массой тела, ожирением, не оцениваем квантильные регрессии), а пока лишь рассматриваем средние значения ИМТ в трех измерениях (возраст, период, когорта), обращая особое внимание на гендерные и когортные различия. 


\section{ЛИТЕРАТУРА}

Аистов А.В. (2018). Доходы респондентов разных поколений. Прикладная эконометрика, $50,23-42$.

Григорьева М.А. (2015). Динамика роста, веса и индекса массы тела реальных поколений россиян 1810-1995 годов в возрасте 18-34 лет. Вестник экономики, права и социологии, Сочииология, (3), 198-202.

Иванова Е.И. (2014). Структурные и социальные факторы здоровья населения России. Вестник РГГУ. Серия «Социологические науки», (4), 138-155.

Колосницына М.Г., Бердникова А.Н. (2009). Избыточный вес: сколько это стоит и что с этим делать? Прикладная эконометрика, 15(3), 72-93.

Колосницына М.Г., Куликова О.А. (2018). Социально-экономические факторы и последствия избыточного веса. Демографическое обозрение, 5(4), 92-124.

Миронов Б. (2003). Рост и вес россиян сталинской эпохи. Демоскоn Weekly, 129-130. URL: http://demoscope.ru/weekly/2003/0129/

Миронов Б. (2012). Благосостояние населения и революции в имперской России: XVIII начало ХХ века. 2-е изд., испр., доп. М.: Весь Мир.

НИУ Высшая школа экономики (2020). Российский мониторинг экономического положения и здоровья населения. Объединенная база данных 1994-2018. Индивиды. URL: https://www.hse.ru/rlms/spss (данные загружены 17.03.2020).

Afshin A., Forouzanfar M.H., Reitsma M.B., Sur P., Estep K., Lee A., ... Murray C.J.L. (2017). Health effects of overweight and obesity in 195 countries over 25 years. New England Journal of Medicine, 377(1), 13-27. DOI: 10.1056/NEJMoa1614362

Arnold M., Leitzmann M., Freisling H., Bray F., Romieu I., Renehan A., Soerjomataram I. (2016). Obesity and cancer: An update of the global impact. Cancer Epidemiology, 41, 8-15. DOI: 10.1016/j.canep.2016.01.003

Badley E.M., Canizares M., Perruccio A.V., Hogg-Johnson S., Gignac M.A. (2015). Benefits gained, benefits lost: Comparing baby boomers to other generations in a longitudinal cohort study of self-rated health. Milbank Quarterly, 93(1), 40-72.

Bhaskaran K., Douglas I., Forbes H., dos Santos-Silva I., Leon D.A., Smeeth L. (2014). Bodymass index and risk of 22 specific cancers: a population-based cohort study of 5.24 million UK adults. The Lancet, 384(9945), 755-65.

Bungum T., Satterwhite M., Jackson A. W., Morrow J., James R. (2003). The relationship of body mass index, medical costs, and job absenteeism. American Journal of Health Behavior, 27(4), 456-462.

Caman O.K., Calling S., Midlov P., Sundquist J., Sundquist K., Johansson S. (2013). Longitudinal age-and cohort trends in body mass index in Sweden - a 24-year follow-up study. BMC Public Health, 13, 893-893.

Castetbon K, Vernay M, Malon A, Salanave B, Deschamps V, Roudier C, Oleko A, Szego E, Hercberg S. (2009). Dietary intake, physical activity and nutritional status in adults: the French nutrition and health survey (ENNS, 2006-2007). British Journal of Nutrition, 102, 733-743.

Cawley J. (2004). The impact of obesity on wages. Journal of Human Resources, 39 (2), 451474. Retrieved from https://ideas.repec.Org/a/uwp/jhriss/v39y2004i2p451-474.html 
Cheng F.W., Gao X., Mitchell D.C., Wood C., Still C.D., Rolston D., Jensen G.L. (2016). Body mass index and all-cause mortality among older adults. Obesity, 24(10), 2232-2239. DOI: 10.1002/oby.21612

Cole T.J., Bellizzi M.C., Flegal K.M., Dietz W.H. (2000). Establishing a standard definition for child overweight and obesity worldwide: international survey. British Medical Journal, 320(7244), 1240-1243. DOI: 10.1136/bmj.320.7244.1240

Conley D., Glauber R. (2007). Gender, body mass, and socioeconomic status: new evidence from the PSID. Advances in health economics and health services research, 17, 253-275.

Danubio M.E., Miranda G., Vinciguerra M.G., Vecchi E., Rufo F. (2008). Comparison of selfreported and measured height and weight: implications for obesity research among young adults. Economics and human biology, 6(1), 181-90.

Dassonneville R. (2013). Questioning generational replacement. An age, period and cohort analysis of electoral volatility in the netherlands, 1971-2010. Electoral Studies, 32(1), 37-47. DOI: https://doi.org/10.1016/j.electstud.2012.09.004

Di Angelantonio E., Bhupathiraju S.N., Wormser D., Gao P., Kaptoge S., Berrington de Gonzalez A., ... Hu F.B. (2016). Body-mass index and all-cause mortality: individualparticipant-data meta-analysis of 239 prospective studies in four continents. The Lancet, 388(10046), 776-786.

Di Cesare M., Bentham J., Stevens G.A., Zhou B., Danaei G., Lu Y., ... NCD Risk Factor Collaboration (2016). Trends in adult body-mass index in 200 countries from 1975 to 2014: a pooled analysis of 1698 population-based measurement studies with 19.2 million participants. Lancet, 387(10026), 1377-1396.

Drøyvold W.B., Nilsen T.I.L., Kriiger O., Holmen T.L., Krokstad S., Midthjell K., Holmen J. (2006). Change in height, weight and body mass index: Longitudinal data from the HUNT study in Norway. International journal of obesity, 30, 935-939.

Ethan F., Christopher W, (2019). Analyzing Age-Period-Cohort Data: A Review and Critique. Annual Review of Sociology, 45(1), 467-492. DOI: https://doi.org/10.1146/annurev-soc073018-022616

Farhat T., Iannotti R.J., Simons-Morton B.G. (2010). Overweight, obesity, youth, and health-risk behaviors. American Journal of Preventive Medicine, 38(3), 258-267.

Fu W. (2016). Constrained estimators and consistency of a regression model on a lexis diagram. Journal of the American Statistical Association, 111(513), 180-199. DOI: 10.1080/01621459.2014.998761

Godina E. (2011). Secular trends in some Russian populations. Anthropologischer Anzeiger, 68(4), 367-377.

Gordon-Larsen P., Wang H., Popkin B.M. (2014). Overweight dynamics in Chinese children and adults. Obesity Reviews, 15(S1), 37-48. DOI: 10.1111/obr.12121

Gu H., Shao S., Liu J., Fan Z., Chen Y., Ni J., ... Wang J. (2019). Age- and sex- associated impacts of body mass index on stroke type risk: A 27 -year prospective cohort study in a lowincome population in China. Frontiers in Neurology, 10, 1-9. DOI:

10.3389/fneur.2019.00456

Gurevich K.G., Poston W.S.C., Anders B., Ivkina M.A., Archangelskaya A., Jitnarin N., Starodubov V.I. (2017). Obesity prevalence and accuracy of BMI-defined obesity in Russian firefighters. Occupational Medicine, 67(1), 61-63. DOI: 10.1093/occmed/kqw136 
Harrington M., Gibson S., Cottrell R.C. (2009). A review and meta-analysis of the effect of weight loss on all-cause mortality risk. Nutrition Research Reviews, 22(1), 93-108. DOI: $10.1017 /$ S0954422409990035

Heo J., Beck A.N., Lin S.-F., Marcelli E., Lindsay S., Karl Finch B. (2018). Cohort-based income gradients in obesity among U.S. adults. American Journal of Human Biology, 30(2), e23084.

Holford T.R. (1983). The estimation of age, period and cohort effects for vital rates. Biometrics, 39(2), 311-324. DOI: 10.1016/0021-9681(85)90106-7

Hosseini M., Kelishadi R., Baikpour M., Ataei N., Qorbani M., Yousefifard M., ... Mohammad K. (2017). Age-period-cohort analysis of obesity and overweight in Iranian children and adolescents. International Journal of Endocrinology and Metabolism, 15(4), e13561. DOI: 10.5812/ijem.13561

Hu Y., Malyutina S., Pikhart H., Peasey A., Holmes M.V., Hubacek J., ... Bobak M. (2017). The relationship between body mass index and 10-year trajectories of physical functioning in middle-aged and older Russians: Prospective results of the Russian HAPIEE study. The Journal of Nutrition, Health and Aging, 21(4), 381-388.

Huffman S.K., Rizov M. (2007). Determinants of obesity in transition economies: The case of Russia. Economics and Human Biology, 5(3), 379-391.

Jaacks L., Gordon-Larsen P., Mayer-Davis E., Adair L., Popkin B. (2013). Age, period and cohort effects on adult body mass index and overweight from 1991 to 2009 in China: the China Health and Nutrition Survey. International journal of epidemiology, 42(3), 828-837.

Jiang T., Gilthorpe M., Shiely F., Harrington J., Perry I., Kelleher C., Tu Y.-K. (2013). Ageperiod-cohort analysis for trends in body mass index in Ireland. BMC Public Health, 13, 889889.

Kalichman L., Livshits G., Kobyliansky E. (2006). Indices of body composition and chronic morbidity: A cross? sectional study of a rural population in central Russia. American Journal of Human Biology, 18(3), 350-358.

Kant A.K., Graubard B.I., Kumanyika S.K. (2007). Trends in Black-White Differentials in Dietary Intakes of U.S. Adults, 1971-2002. American Journal of Preventive Medicine, 32(4), 264-272.e1. DOI:10.1016/j.amepre.2006.12.011

Kawachi A., Shimazu T., Budhathoki S., Sawada N., Yamaji T., Iwasaki M., ... Tsugane S. (2019). Association of BMI and height with the risk of endometrial cancer, overall and by histological subtype: a population-based prospective cohort study in Japan. European Journal of Cancer Prevention, 28(3), 196-202.

Kline B., Tobias J.L. (2008). The wages of BMI: Bayesian analysis of a skewed treatmentresponse model with nonparametric endogeneity. Journal of Applied Econometrics, 23(6), 767-793.

Kolarzyk E., Pac A., Shpakou A., Kleszczewska E., Klimackaya L., Laskiene S. (2012a). Nutrition of overweight and obese students. Central European Journal of Medicine, 7(5), 665-671. DOI: 10.2478/s11536-012-0028-6

Kolarzyk E., Shpakou A., Kleszczewska E., Klimackaya L., Laskiene S. (2012b). Nutritional status and food choices among first year medical students. Central European Journal of Medicine, 7, 396-408. DOI: 10.2478/s11536-011-0167-1

Kozyreva P., Kosolapov M., Popkin B.M. (2016). Data Resource Profile: The Russia Longitudinal Monitoring Survey - Higher School of Economics (RLMS-HSE) Phase II: 
Monitoring the Economic and Health Situation in Russia, 1994-2013. International Journal of Epidemiology, 45(2), 395-401. DOI: https://10.1093/ije/dyv357

Krul A.J., Daanen H.A.M., Choi H. (2010). Self-reported and measured weight, height and body mass index (BMI) in Italy, the Netherlands and North America. The European Journal of Public Health, 21(4), 414-419. DOI:10.1093/eurpub/ckp228

Kuczmarski M. F., Kuczmarski R.J., Najjar M. (2001). Effects of age on validity of selfreported height, weight, and body mass index: Findings from the Third National Health and Nutrition Examination Survey, 1988-1994. Journal of the American Dietetic Association, 101(1), 28-34.

Kwok M.K., Tu Y.K., Kawachi I., Schooling C. M. (2017). Age-period-cohort analysis of trends in blood pressure and body mass index in children and adolescents in Hong Kong. Journal of epidemiology and community health, 71(12), 1161-1167.

Lainscak M., von Haehling S., Doehner W., Anker S. (2012). The obesity paradox in chronic disease: Facts and numbers. Journal of cachexia, sarcopenia and muscle, 3, 1-4. DOI: 10.1007/s13539-012-0059-5

Lambert A.A., Putcha N., Drummond M.B., Boriek A.M., Hanania N.A., Kim V., ... COPDGene Investigators (2017). Obesity is associated with increased morbidity in moderate to severe COPD. CHEST, 151(1), 68-77. DOI: 10.1016/j.chest.2016.08.1432

Ley C. J., Lees B., Stevenson J. C. (1992). Sex- and menopause-associated changes in body-fat distribution. The American Journal of Clinical Nutrition, 55(5), 950-954.

DOI:10.1093/ajen/55.5.950

Maruyama S., Nakamura S. (2018). Why are women slimmer than men in developed countries? Economics and Human Biology, 30, 1-13.

Mason, K. O., Mason, W. M., Winsborough, H. H., \& Poole, W. K. (1973). Some methodological issues in cohort analysis of archival data. American Sociological Review, 38, 242-258.

Mock C.N., Grossman D.C., Kaufman R.P., Mack C.D., Rivara F.P. (2002). The relationship between body weight and risk of death and serious injury in motor vehicle crashes. Accident Analysis and Prevention, 34(2), 221-228.

Must A., Dallal G., Dietz W. (1991a). Reference data for obesity: 85th and 95th percentiles of body mass index (wt/ht2)-a correction. The American Journal of Clinical Nutrition, 54(5), 773-773.

Must A., Dallal G., Dietz W. (1991b). Reference data for obesity: 85th and 95th percentiles of body mass index (wt/ht2) and triceps skinfold thickness. The American Journal of Clinical Nutrition, 53(4), 839-846.

Ng C., Corey P.N., Young T.K. (2012). Divergent body mass index trajectories between aboriginal and non-aboriginal canadians 1994-2009 4an exploration of age, period, and cohort effects. American Journal of Human Biology, 24, 170-176.

Nooyens A.C., Visscher T.L., Verschuren W.M., Schuit A.J., Boshuizen H.C., van Mechelen W., Seidell J.C. (2009). Age, period and cohort effects on body weight and body mass index in adults: The Doetinchem Cohort Study. Public Health Nutrition, 12(6), 862-870. DOI: $10.1017 / \mathrm{S} 1368980008003091$

Ogden C.L., Carroll M.D., Lawman H.G., Fryar C.D., Kruszon-Moran D., Kit B.K., Flegal K.M. (2016). Trends in obesity prevalence among children and adolescents in the United States, 
1988-1994 through 2013-2014. Journal of the American Medical Association, 315(21), 22922299. DOI: $10.1001 /$ jama.2016.6361

Pelzer B., te Grotenhuis M., Eisinga R., Schmidt-Catran A.W. (2015). The nonuniqueness property of the intrinsic estimator in APC models. Demography, 52, 315-327.

Rodriguez C., Freedland S.J., Deka A., Jacobs E.J., McCullough M.L., Patel A.V., ... Calle E.E. (2007). Body mass index, weight change, and risk of prostate cancer in the Cancer Prevention Study II Nutrition Cohort. Cancer Epidemiology and Prevention Biomarkers, 16(1), 63-69.

Rosenquist J., Lehrer S.F., O'Malley A., Zaslavsky A., Smoller J., Christakis N. (2014). Cohort of birth modifies the association between FTO genotype and BMI. Proceedings of the National Academy of Sciences, 112, 354-359.

Roth L.W., Allshouse A.A., Lesh J., Polotsky A.J., Santoro N. (2013). The correlation between self-reported and measured height, weight, and BMI in reproductive age women. Maturitas, $76,185-188$.

StataCorp. (2019a). Stata graphics reference manual: Release 16. College Station, TX: A Stata Press Publication.

StataCorp. (2019b). Stata base reference manual: Release 16. College Station, TX: A Stata Press Publication.

Su S.-Y., Lee W.-C. (2019). Age-period-cohort analysis with a constant-relative-variation constraint for an apportionment of period and cohort slopes. PLOS ONE, 14(12). DOI: 10.1371/journal.pone.0226678

Twig G., Yaniv G., Levine H., Leiba A., Goldberger N., Derazne E., ... Kark J.D. (2016). Bodymass index in 2.3 million adolescents and cardiovascular death in adulthood. The New England Journal of Medicine, 374(25), 2430-2440.

Vandeschrick C. (2001). The Lexis diagram, a misnomer. Demographic Research, 4(3), 97124. DOI: 10.4054/DemRes.2001.4.3

Wahl S., Drong A., Lehne B., Loh M., Scott W.R., Kunze S., ... Chambers J.C. (2017). Epigenome-wide association study of body mass index, and the adverse outcomes of adiposity. Nature, 541(7635), 81-86. DOI: 10.1038/nature20784

Wang Y., Wang J. (2002). A comparison of international references for the assessment of child and adolescent overweight and obesity in different populations. European Journal of Clinical Nutrition, 56(10), 973-982. DOI: 10.1038/sj.ejen.1601415

Wansink B., Cheney M., Chan N. (2003). Exploring comfort food preferences across age and gender1. Physiology \& Behavior, 79(4-5), 739-747. DOI:10.1016/s0031-9384(03)00203-8

Wardle J., Haase A.M., Steptoe A., Nillapun M., Jonwutiwes K., Bellisie F. (2004). Gender differences in food choice: The contribution of health beliefs and dieting. Annals of Behavioral Medicine, 27(2), 107-116. DOI:10.1207/s15324796abm2702_5

WHO (1995). Physical status: the use and interpretation of anthropometry (Tech. Rep.). WHO Technical Report Series No. 854. Geneva: World Health Organization.

Wilson R., Abbott J.H. (2018). Age, period and cohort effects on body mass index in New Zealand, 1997-2038. Australian and New Zealand Journal of Public Health, 42(4), 396-402.

Wooldridge J.M. (2010). Econometric Analysis of Gross Section and Panel Data. Cambridge, Massachusetts; London, England: The MIT Press. 
Yang Y., Land K.C. (2013). Age-period-cohort analysis: New models, methods, and empirical applications. CRC Press.

Yang Y., Land, K.C. (2006). A mixed models approach to the age-period-cohort analysis of repeated cross-section surveys, with an application to data on trends in verbal test scores. Sociological Methodology, 36(1), 75-97. DOI: 10.1111/j.1467-9531.2006.00175.x

Yi S.-W., Ohrr H., Shin S.-A., Yi J.-J. (2015). Sex-age-specific association of body mass index with all-cause mortality among 12.8 million Korean adults: a prospective cohort study. International Journal of Epidemiology, 44(5), 1696-1705. DOI: 10.1093/ije/dyv138

Yu E., Ley S., E Manson J., Willett W., Satija A., B Hu F., Stokes A. (2017). Weight history and all-cause and cause-specific mortality in three prospective cohort studies. Annals of Internal Medicine, 166(9), 613-620.

Zagorsky J.L. (2005). Health and wealth: The late-20th century obesity epidemic in the U.S. Economics and Human Biology, 3(2), 296-313.

Zhang L. (2015). How does age matter in the linkage between religious involvement and adult self-rated health? Advances in Applied Sociology, 5(4), 146-160.

DOI: 10.4236/aasoci.2015.54014. 


\section{ПРИЛОЖЕНИЕ}

\section{Таблица П1. Оценки ИМТ по разным странам}

\begin{tabular}{|c|c|c|c|c|}
\hline Страна; годы & $\begin{array}{c}\text { Авторы; } \\
\text { источник } \\
\text { данных }\end{array}$ & Возраст & $\begin{array}{l}\text { Количество } \\
\text { наблюдений }\end{array}$ & Выборка. Результаты. \\
\hline $\begin{array}{l}\text { Норвегия; } \\
\text { 1984-1986, }\end{array}$ & $\begin{array}{l}\text { (Drøyvold et } \\
\text { 2006); Nord }\end{array}$ & $20+$ & $\begin{array}{c}21565 \\
\text { мужчин, }\end{array}$ & $\begin{array}{c}\text { В течение } 11 \text { лет доля мужчин с ожирением } \\
\text { выросла с } 6,7 \text { до 15,5\%, женщин - с } 11 \text { до }\end{array}$ \\
\hline
\end{tabular}

1995-1997 Trøndelag Health

Study (HUNT)
24337

женщин
$21 \%$. Наибольший процент мужчин с

ожирением наблюдался в возрастной группе 60-69 лет. У женщин этот максимум был в возрасте 70-79 лет в 1984-1986 гг. и 60-

69 лет в 1995-1997 гг. В возрастной группе

20-29 лет доля мужчин с ожирением выросла с 2,9 до $12,4 \%$, женщин - с 3,7 до $11,9 \%$

\begin{tabular}{|c|c|c|c|c|}
\hline $\begin{array}{l}\text { Нидерланды; } \\
1987-2002\end{array}$ & $\begin{array}{l}\text { (Nooyens et al. } \\
\text { 2009); } \\
\text { Doetinchem } \\
\text { Cohort Study }\end{array}$ & $20-59$ & $\begin{array}{c}1988 \\
\text { мужчин, } \\
2082 \\
\text { женщин }\end{array}$ & $\begin{array}{l}\text { ИМТ молодых поколений выше, чем у } \\
\text { старших в том же возрасте }\end{array}$ \\
\hline $\begin{array}{l}\text { США; 1986, } \\
1999,2001\end{array}$ & $\begin{array}{l}\text { (Conley, Glauber } \\
\text { 2007); Panel } \\
\text { Study of Income } \\
\text { Dynamics } \\
\text { (PSID) }\end{array}$ & $25+$ & 2322 & $\begin{array}{c}\text { Исследование близнецов. Среднее значение } \\
\text { ИМТ мужчин в } 1986 \text { г. равно } 25,82, \\
\text { женщин }-24,20\end{array}$ \\
\hline $\begin{array}{l}\text { США; } 1971- \\
2008\end{array}$ & $\begin{array}{l}\text { (Rosenquist et al. } \\
\text { 2014); Offspring } \\
\text { Cohort, } \\
\text { Framingham } \\
\text { Heart Study } \\
\text { (FHS) }\end{array}$ & $27-63$ & 5124 & $\begin{array}{c}\text { Монотонный рост ИМТ, начиная со } \\
\text { значений 24,5-26,5 у группы респондентов } \\
\text { 35-40 лет, до 27-29 у 55-60-летних. } \\
\text { Возрастные профили ИМТ респондентов, } \\
\text { родившихся после } 1942 \text { г., лежат на 1-2 } \\
\text { единицы ИМТ выше, чем у респондентов, } \\
\text { родившихся до } 1942 \text { г. }\end{array}$ \\
\hline $\begin{array}{l}\text { США; } 1988- \\
2014\end{array}$ & $\begin{array}{l}\text { (Ogden et al. } \\
\text { 2016); National } \\
\text { Health and } \\
\text { Nutrition } \\
\text { Examination } \\
\text { Survey } \\
\text { (NHANES) }\end{array}$ & $2-19$ & 40780 & $\begin{array}{c}\text { Среди подростков 12-19 лет ожирение } \\
\text { выросло с 10,5\%, наблюдавшихся в 1988- } \\
1994 \text { гг., до 20,6\% - в 2013-2014 гг. Процент } \\
\text { детей с ожирением в возрасте 2-5 лет рос до } \\
\text { 2003-2004 гг., после этого снижался. } \\
\text { В группе 6-11-летних процент респондентов } \\
\text { с ожирением рос до 2007-2008 гг., после } \\
\text { этого не менялся }\end{array}$ \\
\hline $\begin{array}{l}\text { США; 1971- } \\
2012\end{array}$ & $\begin{array}{l}\text { (Heo et al. 2018); } \\
\text { NHANES }\end{array}$ & $18-80$ & 56820 & $\begin{array}{c}\text { Процент женщин с ожирением выше, чем } \\
\text { мужчин. Респонденты, родившиеся до } \\
1930 \text { г., менее склонны к ожирению по срав- } \\
\text { нению молодыми поколениями }\end{array}$ \\
\hline $\begin{array}{l}\text { Канада; } \\
\text { 1994-2009 }\end{array}$ & $\begin{array}{l}\text { (Ng et al. 2012); } \\
\text { National } \\
\text { Population } \\
\text { Health Survey }\end{array}$ & $5-68$ & 11278 & $\begin{array}{c}\text { Линейные регрессионные модели } \\
\text { предсказали высокие ИМТ и темпы роста } \\
\text { ИМТ с возрастом у молодых когорт }\end{array}$ \\
\hline $\begin{array}{l}\text { Швеция; } \\
\text { 1980-2005 }\end{array}$ & $\begin{array}{l}\text { (Caman et al. } \\
\text { 2013); Swedish } \\
\text { Annual Level of } \\
\text { Living Survey } \\
\text { (SALLS) }\end{array}$ & $16-71$ & $\begin{array}{c}2728 \\
\text { мужчин, } \\
2770 \\
\text { женщин }\end{array}$ & $\begin{array}{c}\text { Средний ИМТ мужчин вырос за время } \\
\text { наблюдений с } 24,1 \text { до 25,5, женщин - с } 23,1 \\
\text { до 24,3. Наибольшие ежегодные темпы роста } \\
\text { ИМТ наблюдались у молодых когорт }\end{array}$ \\
\hline $\begin{array}{l}\text { Китай; 1991- } \\
2009\end{array}$ & $\begin{array}{c}\text { (Jaacks et al. } \\
\text { 2013); China } \\
\text { Health and } \\
\text { Nutrition Survey } \\
\text { (CHNS) }\end{array}$ & $22-60$ & 53298 & $\begin{array}{c}\text { До } 2006 \text { г. процент женщин с избыточной } \\
\text { массой тела был выше, чем мужчин; в } 2006 \text { и } \\
2009 \text { г. доля мужчин с избыточной массой } \\
\text { тела стала выше, чем женщин. ИМТ } \\
\text { молодых когорт выше по сравнению со } \\
\text { старшими поколениями }\end{array}$ \\
\hline
\end{tabular}




\begin{tabular}{|c|c|c|c|c|}
\hline Страна; годы & $\begin{array}{c}\text { Авторы; } \\
\text { источник } \\
\text { данных }\end{array}$ & Возраст & $\begin{array}{l}\text { Количество } \\
\text { наблюдений }\end{array}$ & Выборка. Результаты. \\
\hline $\begin{array}{l}\text { Китай; 1991- } \\
2011\end{array}$ & $\begin{array}{c}\text { (Gordon-Larsen } \\
\text { et al. 2014); } \\
\text { CHNS }\end{array}$ & $18-66$ & 53298 & $\begin{array}{c}\text { Средний ИМТ респондентов 18-65 лет вырос } \\
\text { с 21,5 в } 1991 \text { г. до 22,9 в } 2011 \text { г.; число } \\
\text { женщин с избыточной массой тела выросло с } \\
12,5 \text { до 22,2\%, мужчин - с } 8,0 \text { до } 30,2 \% . \\
\text { Профили ИМТ-возраст молодых поколений } \\
\text { расположены выше предыдущих поколений }\end{array}$ \\
\hline $\begin{array}{l}\text { Гонконг; } \\
1996-2014\end{array}$ & $\begin{array}{c}\text { (Kwok et al. } \\
\text { 2017); Student } \\
\text { Health Service of } \\
\text { the Department } \\
\text { of Health }\end{array}$ & $6-18$ & $\begin{array}{c}196299 \\
\text { мальчиков, } \\
205741 \\
\text { девочек }\end{array}$ & $\begin{array}{c}\text { ИМТ мальчиков монотонно рос с возрастом, } \\
\text { у девочек ИМТ рос до } 16 \text { лет, в возрасте 16- } \\
18 \text { лет практически не менялся. Средний } \\
\text { ИМТ монотонно рос с } 1996 \text { по } 2014 \text { г. } \\
\text { Максимальный ИМТ наблюдался у } \\
\text { поколения 1981-1982 гг. рождения, } \\
\text { минимальный - у родившихся в 2004- } \\
2005 \text { гг. }\end{array}$ \\
\hline
\end{tabular}

\begin{tabular}{|c|c|c|c|c|}
\hline $\begin{array}{l}\text { Иран; 1990- } \\
2011\end{array}$ & $\begin{array}{l}\text { (Hosseini et al. } \\
2017) ; \text { кросс- } \\
\text { секционные } \\
\text { наблюдения (2 } \\
\text { национальных } \\
\text { опроса и } 3 \\
\text { CASPIAN) }\end{array}$ & $<19$ & 80698 & $\begin{array}{c}\text { Процент детей с ожирением снижался с } \\
\text { возрастом. Этот процент рос с } 1990 \text { по } \\
2009 \text { г., начиная с } 2009 \text { г. он перестал } \\
\text { изменяться на выборке мальчиков и } \\
\text { значительно снизился среди девочек. } \\
\text { Процент детей с ожирением мало менялся в } \\
\text { выборке старших когорт, но резко вырос у } \\
\text { детей, рожденных в 1986-1990 гг. }\end{array}$ \\
\hline $\begin{array}{l}\text { Новая } \\
\text { Зеландия; } \\
1997-2015\end{array}$ & $\begin{array}{c}\text { (Wilson, Abbott } \\
\text { 2018); New } \\
\text { Zealand Health } \\
\text { Survey (NZHS), } \\
\text { Adult Nutrition } \\
\text { Survey, National } \\
\text { Nutrition Survey, } \\
\text { Life in New } \\
\text { Zealand survey, } \\
\text { National Diet } \\
\text { Survey }\end{array}$ & $18-89$ & 76294 & $\begin{array}{c}76294 \text { респондентов 18-89 лет. Средине } \\
\text { значения ИМТ выросли с 26,4 в } 1997 \text { г. до } \\
28,3 \text { в } 2015 \text { г. Выявлены ежегодные } \\
\text { линейные тренды, эффекты когорт } \\
\text { статистически не значимы. В предположе- } \\
\text { нии квадратичной зависимости ИМТ от } \\
\text { возраста максимум ИМТ получен в возрасте } \\
55 \text { лет }\end{array}$ \\
\hline $\begin{array}{l}\text { Ирландия; } \\
1998,2002, \\
2007\end{array}$ & $\begin{array}{l}\text { (Jiang et al. } \\
\text { 2013); Surveys } \\
\text { of Lifestyle, } \\
\text { Attitudes and } \\
\text { Nutrition } \\
\text { (SLAN) }\end{array}$ & $18-75$ & $\begin{array}{c}7796 \\
\text { мужчин, } \\
10220 \\
\text { женщин }\end{array}$ & $\begin{array}{c}\text { Выявлены тенденции роста ИМТ с } \\
\text { возрастом и календарным годом. ИМТ } \\
\text { растет у женщин до } 59 \text { лет, затем начинает } \\
\text { снижаться. У мужчин ИМТ растет до } 35 \text { лет, } \\
\text { после этого наблюдается плато и происходит } \\
\text { падение после } 60 \text { лет. ИМТ молодых когорт } \\
\text { ниже по сравнению со старшими } \\
\text { поколениями } \\
\end{array}$ \\
\hline $\begin{array}{l}\text { Япония; } \\
1975-2010\end{array}$ & $\begin{array}{l}\text { (Maruyama, } \\
\text { Nakamura 2018); } \\
\text { Japanese } \\
\text { National } \\
\text { Nutrition Survey } \\
\text { (NNS) }\end{array}$ & $18-59$ & 245880 & $\begin{array}{c}\text { Наблюдается ежегодный рост ИМТ мужчин } \\
\text { и снижение ИМТ женщин. У мужчин 18- } \\
39 \text { лет ИМТ выше, чем у женщин. ИМТ 40- } \\
\text { 49-летних мужчин был выше, чем у женщин, } \\
\text { до } 1980 \text { г., позже соотношение изменилось. } \\
\text { Для респондентов 50-59 лет такой границей } \\
\text { был } 1990 \text { г. }\end{array}$ \\
\hline $\begin{array}{l}\text { Россия; } \\
1994,1999, \\
2002\end{array}$ & $\begin{array}{l}\text { (Kalichman, } \\
\text { Livshits, } \\
\text { Kobyliansky } \\
\text { 2006); Chuvasha } \\
\text { Skeletal Aging } \\
\text { Study (ChuSAS) } \\
\end{array}$ & $18-90$ & $\begin{array}{l}787 \text { мужчин } \\
716 \text { женщин }\end{array}$ & $\begin{array}{c}\text { В сельской местности Чувашии среднее } \\
\text { значение ИМТ мужчин равно 23,2 (пределы } \\
\text { изменений: 15,5-36,4), женщин - 25,2 (15,3- } \\
\text { 44,5). Ожирение наблюдалось у } 3,6 \% \\
\text { мужчин и } 16 \% \text { женщин }\end{array}$ \\
\hline $\begin{array}{l}\text { Россия; } \\
\text { 1994, } 2004\end{array}$ & $\begin{array}{l}\text { (Huffman, Rizov } \\
\text { 2007); RLMS- } \\
\text { HSE }\end{array}$ & $18+$ & $\begin{array}{c}2532 \\
\text { мужчин } \\
3892 \\
\text { женщин }\end{array}$ & $\begin{array}{c}\text { Средний ИМТ равен 26,2 (9,5\% мужчин и } \\
27,8 \% \text { женщин с ожирением) в } 1994 \text { г. и } 27,4 \\
\text { (16,3\% мужчин и } 36,6 \% \text { женщин с } \\
\text { ожирением) в } 2004 \text { г. }\end{array}$ \\
\hline
\end{tabular}




\begin{tabular}{|c|c|c|c|c|}
\hline Страна; годы & $\begin{array}{l}\text { Авторы; } \\
\text { источник } \\
\text { данных }\end{array}$ & Возраст & $\begin{array}{l}\text { Количество } \\
\text { наблюдений }\end{array}$ & Выборка. Результаты. \\
\hline $\begin{array}{l}\text { Россия; } \\
2006,1996- \\
2006\end{array}$ & $\begin{array}{l}\text { (Колосницына, } \\
\text { Бердникова } \\
\text { 2009); RLMS- } \\
\text { HSE }\end{array}$ & $18+$ & $\begin{array}{c}4803 \\
\text { мужчин } \\
6462 \\
\text { женщин }\end{array}$ & $\begin{array}{c}\text { Средний ИМТ мужчин в } 2006 \text { г. равен 25,08 } \\
\text { (12,33\% с ожирением), женщин - 26,28 } \\
\text { (24,77\% с ожирением). Максимальный ИМТ } \\
\text { наблюдался в группе возрастов } 60-69 \text { лет } \\
\text { (26,51 у мужчин, } 29,14 \text { у женщин). } \\
\text { Графические иллюстрации показывают } \\
\text { положительную динамику ИМТ, долей } \\
\text { людей с избыточной массой тела и } \\
\text { ожирением с } 1996 \text { по } 2006 \text { г. }\end{array}$ \\
\hline Россия; 2004 & $\begin{array}{l}\text { (Godina 2011); } \\
\text { Институт и } \\
\text { музей } \\
\text { антропологии } \\
\text { МГУ }\end{array}$ & $7-17$ & 1400 & $\begin{array}{c}\text { В г. Саратов. ИМТ девочек 15-17 лет } \\
\text { примерно на } 2 \text { единицы ниже по сравнению } \\
\text { с данными } 1959 \text { и } 1969 \text { г. Для мальчиков и } \\
\text { девочек } 7 \text { лет ИМТ примерно на } 1 \text { единицу } \\
\text { выше исторических данных }\end{array}$ \\
\hline $\begin{array}{l}\text { Россия; } \\
1828-2012\end{array}$ & $\begin{array}{l}\text { (Григорьева } \\
\text { 2015); RLMS- } \\
\text { HSE и работы } \\
\text { (Миронов 2003; } \\
\text { 2012) }\end{array}$ & $18+$ & Неизвестно & $\begin{array}{c}\text { ИМТ мужчин в возрасте 18-34 лет растет } \\
\text { при переходе от } 1810 \text { к } 1900 \text { г. рождения. } \\
\text { Согласно оценкам на данных RLMS-HSE } \\
\text { средний ИМТ населения монотонно растет с } \\
1994 \text { по } 2012 \text { г. Основной вклад в долю } \\
\text { респондентов с избыточной массой тела и } \\
\text { ожирением дают мужчины и женщины 35- } \\
54 \text { лет } \\
\end{array}$ \\
\hline $\begin{array}{l}\text { Россия; } \\
\text { 2002-2012 }\end{array}$ & $\begin{array}{c}\text { (Hu et al. 2017); } \\
\text { Health, Alcohol } \\
\text { and Psychosocial } \\
\text { factors In Eastern } \\
\text { Europe } \\
\text { (HAPIEE) }\end{array}$ & $45-69$ & 9222 & $\begin{array}{c}\text { Ожирение наблюдалось у 21\% мужчин и } \\
47,4 \% \text { женщин }\end{array}$ \\
\hline Россия; 2015 & $\begin{array}{l}\text { (Gurevich et al. } \\
\text { 2017); } \\
\text { медицинский } \\
\text { осмотр }\end{array}$ & $\begin{array}{c}\text { Срединй } \\
\text { возраст } \\
39,9 \pm \\
11,2\end{array}$ & 167 & $\begin{array}{c}\text { Среди пожарных Московского региона } \\
\text { избыточная масса тела и ожирение } \\
\text { наблюдалось у } 69 \% \text { респондентов }\end{array}$ \\
\hline $\begin{array}{l}\text { Россия; } \\
1995-2016\end{array}$ & $\begin{array}{l}\text { (Колосницына, } \\
\text { Куликова 2018); } \\
\text { RLMS-HSE }\end{array}$ & $19-100$ & $\begin{array}{c}22516 \\
\text { мужчин, } \\
31999 \\
\text { женщин }\end{array}$ & $\begin{array}{c}\text { Наблюдается ежегодный рост ИМТ. ИМТ } \\
\text { женщин выше, чем у мужчин. В } 2016 \text { г. } \\
62,2 \% \text { респондентов имели избыточную } \\
\text { массу тела }\end{array}$ \\
\hline
\end{tabular}


Таблица 2. Число респондентов с информацией о ИМТ

\begin{tabular}{lccc}
\hline Год & Женщины & Мужчины & Всего \\
\hline 1994 & 4226 & 3401 & 7627 \\
1995 & 3724 & 3032 & 6756 \\
1996 & 3507 & 2812 & 6319 \\
1998 & 3756 & 3054 & 6810 \\
2000 & 3974 & 3104 & 7078 \\
2001 & 4500 & 3397 & 7897 \\
2002 & 4823 & 3685 & 8508 \\
2003 & 4718 & 3584 & 8302 \\
2004 & 4811 & 3572 & 8383 \\
2005 & 4567 & 3456 & 8023 \\
2006 & 6132 & 4567 & 10699 \\
2007 & 6105 & 4574 & 10679 \\
2008 & 5951 & 4376 & 10327 \\
2009 & 5967 & 4392 & 10359 \\
2010 & 9152 & 6781 & 15933 \\
2011 & 9200 & 6746 & 15946 \\
2012 & 9683 & 7037 & 16720 \\
2013 & 9288 & 6823 & 13550 \\
2014 & 7741 & 5809 & 13498 \\
2015 & 7756 & 5742 & 13706 \\
2016 & 7920 & 5786 & 13786 \\
2017 & 7928 & 5858 & 13354 \\
2018 & 7711 & 5643 & 250371 \\
Всего & 143140 & 107231 & \\
\hline
\end{tabular}

Источник: Расчеты авторов на основе данных RLMS-HSE(НИУ Высшая школа... 2020).

Таблица 3. Оценки параметров модели (1)

\begin{tabular}{|c|c|c|}
\hline & Мужчины & Женщины \\
\hline \multicolumn{3}{|c|}{ Возраст (18 лет - базовая категория) } \\
\hline \multirow[t]{2}{*}{19} & $0,406^{* * *}$ & $0,156^{* *}$ \\
\hline & $(0,068)$ & $(0,071)$ \\
\hline \multirow[t]{2}{*}{20} & $0,680^{* * *}$ & $0,367^{* * *}$ \\
\hline & $(0,069)$ & $(0,073)$ \\
\hline \multirow[t]{2}{*}{21} & $0,904^{* * *}$ & $0,520^{* * *}$ \\
\hline & $(0,071)$ & $(0,075)$ \\
\hline \multirow[t]{2}{*}{22} & $1,026^{* * *}$ & $0,782^{* * *}$ \\
\hline & $(0,074)$ & $(0,078)$ \\
\hline \multirow[t]{2}{*}{23} & $1,315^{* * *}$ & $1,099^{* * *}$ \\
\hline & $(0,077)$ & $(0,081)$ \\
\hline \multirow[t]{2}{*}{24} & $1,465^{* * *}$ & $1,265^{* * *}$ \\
\hline & $(0,080)$ & $(0,083)$ \\
\hline \multirow[t]{2}{*}{25} & $1,630^{* * *}$ & $1,494^{* * *}$ \\
\hline & $(0,084)$ & $(0,087)$ \\
\hline \multirow[t]{2}{*}{26} & $1,783^{* * *}$ & $1,729^{* * *}$ \\
\hline & $(0,087)$ & $(0,091)$ \\
\hline \multirow[t]{2}{*}{27} & $1,929^{* * *}$ & $1,932^{* * *}$ \\
\hline & $(0,092)$ & $(0,095)$ \\
\hline \multirow[t]{2}{*}{28} & $2,104^{* * *}$ & $2,134^{* * *}$ \\
\hline & $(0,096)$ & $(0,099)$ \\
\hline \multirow[t]{2}{*}{29} & $2,349^{* * * *}$ & $2,308^{* * *}$ \\
\hline & $(0,101)$ & $(0,103)$ \\
\hline \multirow[t]{2}{*}{30} & $2,485^{* * *}$ & $2,546^{* * *}$ \\
\hline & $(0,106)$ & $(0,108)$ \\
\hline \multirow[t]{2}{*}{31} & $2,535^{* * *}$ & $2,780^{* * * *}$ \\
\hline & $(0,112)$ & $(0,113)$ \\
\hline 32 & $2,660^{* * * *}$ & $3,052^{* * *}$ \\
\hline
\end{tabular}




\begin{tabular}{|c|c|c|}
\hline & Мужчины & Женщины \\
\hline & $(0,117)$ & $(0,118)$ \\
\hline \multirow[t]{2}{*}{33} & $2,726^{* * * *}$ & $3,190^{* * *}$ \\
\hline & $(0,123)$ & $(0,123)$ \\
\hline \multirow[t]{2}{*}{34} & $2,863^{* * * *}$ & $3,363^{* * *}$ \\
\hline & $(0,128)$ & $(0,128)$ \\
\hline \multirow[t]{2}{*}{35} & $2,934^{* * *}$ & $3,547^{* * *}$ \\
\hline & $(0,134)$ & $(0,133)$ \\
\hline \multirow[t]{2}{*}{36} & $3,048^{* * *}$ & $3,749^{* * *}$ \\
\hline & $(0,139)$ & $(0,139)$ \\
\hline \multirow[t]{2}{*}{37} & $3,182^{* * *}$ & $3,951^{* * *}$ \\
\hline & $(0,145)$ & $(0,145)$ \\
\hline \multirow[t]{2}{*}{38} & $3,254^{* * * *}$ & $4,102^{* * *}$ \\
\hline & $(0,150)$ & $(0,150)$ \\
\hline \multirow[t]{2}{*}{39} & $3,274^{* * *}$ & $4,296^{* * *}$ \\
\hline & $(0,156)$ & $(0,155)$ \\
\hline \multirow[t]{2}{*}{40} & $3,399^{* * *}$ & $4,497^{* * *}$ \\
\hline & $(0,162)$ & $(0,161)$ \\
\hline \multirow[t]{2}{*}{41} & $3,389^{* * *}$ & $4,745^{* * *}$ \\
\hline & $(0,168)$ & $(0,166)$ \\
\hline \multirow[t]{2}{*}{42} & $3,524^{* * * *}$ & $4,874^{* * *}$ \\
\hline & $(0,174)$ & $(0,172)$ \\
\hline \multirow[t]{2}{*}{43} & $3,472^{* * *}$ & $5,066^{* * *}$ \\
\hline & $(0,180)$ & $(0,177)$ \\
\hline \multirow[t]{2}{*}{44} & $3,590^{* * *}$ & $5,249^{* * *}$ \\
\hline & $(0,186)$ & $(0,183)$ \\
\hline \multirow[t]{2}{*}{45} & $3,599^{* * *}$ & $5,421^{* * *}$ \\
\hline & $(0,192)$ & $(0,189)$ \\
\hline \multirow[t]{2}{*}{46} & $3,677^{* * *}$ & $5,591^{* * * *}$ \\
\hline & $(0,197)$ & $(0,195)$ \\
\hline \multirow[t]{2}{*}{47} & $3,692^{* * *}$ & $5,784^{* * *}$ \\
\hline & $(0,204)$ & $(0,200)$ \\
\hline \multirow[t]{2}{*}{48} & $3,687^{* * *}$ & $6,078^{* * *}$ \\
\hline & $(0,209)$ & $(0,206)$ \\
\hline \multirow[t]{2}{*}{49} & $3,739^{* * *}$ & $6,177^{* * *}$ \\
\hline & $(0,215)$ & $(0,212)$ \\
\hline 50 & $3,804^{* * *}$ & $6,285^{* * *}$ \\
\hline & $(0,222)$ & $(0,217)$ \\
\hline 51 & $3,844^{* * *}$ & $6,498^{* * *}$ \\
\hline & $(0,228)$ & $(0,223)$ \\
\hline 52 & $3,857^{* * *}$ & $6,647^{* * *}$ \\
\hline & $(0,234)$ & $(0,229)$ \\
\hline 53 & $3,857^{* * *}$ & $6,819^{* * *}$ \\
\hline & $(0,241)$ & $(0,235)$ \\
\hline 54 & $3,930^{* * * *}$ & $6,978^{* * *}$ \\
\hline & $(0,247)$ & $(0,241)$ \\
\hline 55 & $3,886^{* * *}$ & $7,139^{* * *}$ \\
\hline & $(0,253)$ & $(0,247)$ \\
\hline 56 & $3,867^{* * *}$ & $7,267^{* * *}$ \\
\hline & $(0,259)$ & $(0,254)$ \\
\hline 57 & $3,932^{* * *}$ & $7,359^{* * *}$ \\
\hline & $(0,266)$ & $(0,260)$ \\
\hline 58 & $3,961^{* * * *}$ & $7,433^{* * *}$ \\
\hline & $(0,272)$ & $(0,266)$ \\
\hline 59 & $3,925^{* * *}$ & $7,502^{* * *}$ \\
\hline & $(0,278)$ & $(0,272)$ \\
\hline 60 & $3,961^{* * * *}$ & $7,575^{* * *}$ \\
\hline & $(0,284)$ & $(0,277)$ \\
\hline 61 & $3,981^{* * * *}$ & $7,641^{* * *}$ \\
\hline & $(0,290)$ & $(0,283)$ \\
\hline 62 & $3,886^{* * *}$ & $7,684^{* * * *}$ \\
\hline
\end{tabular}




\begin{tabular}{|c|c|c|}
\hline & Мужчины & Женщины \\
\hline & $(0,297)$ & $(0,290)$ \\
\hline \multirow[t]{2}{*}{63} & $3,979^{* * *}$ & $7,695^{* * *}$ \\
\hline & $(0,303)$ & $(0,296)$ \\
\hline \multirow[t]{2}{*}{64} & $3,954^{* * * *}$ & $7,804^{* * * *}$ \\
\hline & $(0,310)$ & $(0,302)$ \\
\hline \multirow[t]{2}{*}{65} & $3,967^{* * *}$ & $7,793^{* * *}$ \\
\hline & $(0,316)$ & $(0,308)$ \\
\hline \multirow[t]{2}{*}{66} & $3,984^{* * *}$ & $7,835^{\text {*** }}$ \\
\hline & $(0,323)$ & $(0,314)$ \\
\hline \multirow[t]{2}{*}{67} & $3,863^{* * *}$ & $7,813^{* * *}$ \\
\hline & $(0,329)$ & $(0,320)$ \\
\hline \multirow[t]{2}{*}{68} & $3,905^{* * * *}$ & $7,883^{* * * *}$ \\
\hline & $(0,336)$ & $(0,327)$ \\
\hline \multirow[t]{2}{*}{69} & $3,872^{* * *}$ & $7,885^{* * *}$ \\
\hline & $(0,342)$ & $(0,332)$ \\
\hline \multirow[t]{2}{*}{70} & $3,894^{* * *}$ & $7,976^{* * *}$ \\
\hline & $(0,348)$ & $(0,339)$ \\
\hline \multirow[t]{2}{*}{71} & $3,919^{* * *}$ & $7,810^{* * * *}$ \\
\hline & $(0,355)$ & $(0,345)$ \\
\hline \multirow[t]{2}{*}{72} & $3,809^{* * *}$ & $7,785^{* * *}$ \\
\hline & $(0,361)$ & $(0,351)$ \\
\hline \multirow[t]{2}{*}{73} & $3,637^{* * *}$ & $7,741^{* * *}$ \\
\hline & $(0,368)$ & $(0,357)$ \\
\hline \multirow[t]{2}{*}{74} & $3,572^{* * *}$ & $7,807^{* * *}$ \\
\hline & $(0,375)$ & $(0,363)$ \\
\hline \multirow[t]{2}{*}{75} & $3,655^{* * *}$ & $7,773^{* * *}$ \\
\hline & $(0,383)$ & $(0,370)$ \\
\hline \multirow[t]{2}{*}{76} & $3,459^{* * *}$ & $7,754^{* * *}$ \\
\hline & $(0,390)$ & $(0,376)$ \\
\hline \multirow[t]{2}{*}{77} & $3,213^{* * *}$ & $7,639^{* * *}$ \\
\hline & $(0,398)$ & $(0,382)$ \\
\hline \multirow[t]{2}{*}{78} & $3,015^{* * *}$ & $7,637^{* * * *}$ \\
\hline & $(0,405)$ & $(0,389)$ \\
\hline \multirow[t]{2}{*}{79} & $2,971^{* * *}$ & $7,683^{* * *}$ \\
\hline & $(0,413)$ & $(0,396)$ \\
\hline 80 & $2,934^{* * *}$ & $7,723^{* * *}$ \\
\hline & $(0,422)$ & $(0,403)$ \\
\hline 81 & $2,951^{* * * *}$ & $7,650^{* * * *}$ \\
\hline & $(0,434)$ & $(0,412)$ \\
\hline & Год (1994 и 2002 - базовые ка & \\
\hline 1995 & $-0,128^{* * *}$ & $-0,038$ \\
\hline & $(0,048)$ & $(0,049)$ \\
\hline 1996 & $-0,080^{*}$ & $0,097^{* *}$ \\
\hline & $(0,048)$ & $(0,047)$ \\
\hline 1998 & $-0,075^{*}$ & $-0,000$ \\
\hline & $(0,044)$ & $(0,044)$ \\
\hline 2000 & $-0,121^{* * *}$ & $-0,177^{* * *}$ \\
\hline & $(0,045)$ & $(0,044)$ \\
\hline 2001 & $-0,160^{* * *}$ & $-0,172^{* * *}$ \\
\hline & $(0,045)$ & $(0,043)$ \\
\hline 2003 & 0,080 & $0,133^{* * *}$ \\
\hline & $(0,049)$ & $(0,048)$ \\
\hline 2004 & $0,164^{* * *}$ & 0,053 \\
\hline & $(0,054)$ & $(0,052)$ \\
\hline 2005 & $0,222^{* * *}$ & $0,157^{* * *}$ \\
\hline & $(0,058)$ & $(0,057)$ \\
\hline 2006 & $0,444^{* * *}$ & 0,022 \\
\hline & $(0,061)$ & $(0,059)$ \\
\hline 2007 & $0,587^{* * *}$ & 0,002 \\
\hline & $(0,067)$ & $(0,064)$ \\
\hline
\end{tabular}




\begin{tabular}{|c|c|c|}
\hline & Мужчины & Женщины \\
\hline \multirow[t]{2}{*}{2008} & $0,635^{* * *}$ & $-0,000$ \\
\hline & $(0,072)$ & $(0,070)$ \\
\hline \multirow[t]{2}{*}{2009} & $0,637^{* * * *}$ & $-0,012$ \\
\hline & $(0,078)$ & $(0,075)$ \\
\hline \multirow{2}{*}{2010} & $0,749^{* * * *}$ & $-0,064$ \\
\hline & $(0,082)$ & $(0,079)$ \\
\hline \multirow[t]{2}{*}{2011} & $1,009^{* * * *}$ & $0,318^{* * *}$ \\
\hline & $(0,088)$ & $(0,085)$ \\
\hline \multirow[t]{2}{*}{2012} & $0,995^{* * * *}$ & $0,244^{* * * *}$ \\
\hline & $(0,094)$ & $(0,091)$ \\
\hline \multirow[t]{2}{*}{2013} & $1,106^{* * * *}$ & $0,190^{* *}$ \\
\hline & $(0,100)$ & $(0,097)$ \\
\hline \multirow[t]{2}{*}{2014} & $1,185^{* * * *}$ & $0,181^{*}$ \\
\hline & $(0,106)$ & $(0,103)$ \\
\hline \multirow[t]{2}{*}{2015} & $1,177^{* * * *}$ & 0,084 \\
\hline & $(0,112)$ & $(0,109)$ \\
\hline \multirow[t]{2}{*}{2016} & $1,131^{* * * *}$ & $-0,022$ \\
\hline & $(0,119)$ & $(0,115)$ \\
\hline \multirow[t]{2}{*}{2017} & $1,156^{* * * *}$ & $-0,084$ \\
\hline & $(0,125)$ & $(0,121)$ \\
\hline \multirow[t]{2}{*}{2018} & $1,231^{* * * *}$ & $-0,081$ \\
\hline & $(0,131)$ & $(0,127)$ \\
\hline \multirow{2}{*}{ Константа } & $21,944^{* * * *}$ & $21,643^{* * * *}$ \\
\hline & $(0,114)$ & $(0,131)$ \\
\hline Наблюдения & 107231 & 143140 \\
\hline Группы & 19585 & 23443 \\
\hline$R^{2}$ within & 0,111 & 0,115 \\
\hline$R^{2}$ between & 0,125 & 0,279 \\
\hline$R^{2}$ overall & 0,103 & 0,223 \\
\hline$F$ & $130,1^{* * * *}$ & $185,9^{* * * *}$ \\
\hline$H_{o}: u_{i}=0$ & $20,0^{* * *}$ & $30,1^{* * *}$ \\
\hline
\end{tabular}

Примечания: * - Значим на 10\%-ном уровне значимости, ** - значим на 5\%-ном уровне значимости, *** - значим на 1\%-ном уровне значимости. В скобках указаны стандартные ошибки.

Источник: Расчеты авторов на основе данных RLMS-HSE(НИУ Высшая школа... 2020). 


\title{
BODY MASS INDEX DYNAMICS OF RUSSIAN MEN AND WOMEN: AGE-PERIOD-COHORT ANALYSIS
}

\author{
ANDREY AISTOV, EKATERINA ALEKSANDROVA, FARIDA GARIPOVA
}

\begin{abstract}
Using the Russia Longitudinal Monitoring survey (RLMS-HSE) 1994-2018 thes paper presents assessments of the individual dynamics of body mass index (BMI) of Russian men and women for 1994-2018. The panel data made it possible to assess the individual dynamics of respondents' BMI without a significant contribution of the cross-sectional component to the age profile of BMI that could take place within the framework of some age-period-cohort econometric models. Empirical estimates revealed a monotonic increase in the BMI of men in 2001-2011. We show that the BMI of women grows during their life up to 60 years old, and that of men - up to about 40 years old. It was also revealed that there is a tendency for the growth of BMI in younger generations of men. For women, this growth is replaced by a decline that began with the generation of women born in 1950. The estimates of the BMI profiles of men and women in three dimensions (age-period-cohort) obtained in this paper can serve the purposes of improving policy in the field of public health protection.
\end{abstract}

Key words: overweight, public health, Russia, panel data, RLMS-HSE.

Andrey Aistov (aaistov@hse.ru), NATIONAL Research University Higher SchOol of Economics, Russia.

EKATERINA Aleksandrova (ea.aleksandrova@hse.ru), NATIONAL RESEARCh University Higher SchOOL OF ECONOMICS, RUSSIA.

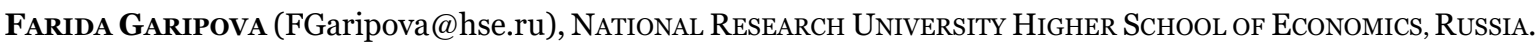

THIS RESEARCH WAS SUPPORTED BY A RUSSIAN SCIENCE FOUNDATION GRANT, PROJECT No20-18-00307, "HEALTH OF

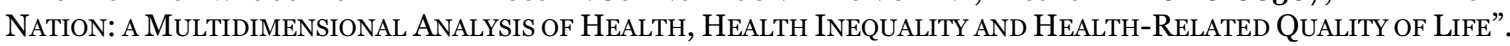

DATE RECEIVED : JANUARY 2021.

\section{REFERENCES}

Afshin A., Forouzanfar M.H., Reitsma M.B., Sur P., Estep K., Lee A., ... Murray C.J.L. (2017). Health effects of overweight and obesity in 195 countries over 25 years. New England Journal of Medicine, 377(1), 13-27. DOI: 10.1056/NEJMoa1614362

Aistov A. 2018. Age-earnings profiles of different generations. Applied Econometrics, Russian Presidential Academy of National Economy and Public Administration (RANEPA), 50, 23 42. (In Russ.).

Arnold M., Leitzmann M., Freisling H., Bray F., Romieu I., Renehan A., Soerjomataram I. (2016). Obesity and cancer: An update of the global impact. Cancer Epidemiology, 41, 8-15. DOI: 10.1016/j.canep.2016.01.003

Badley E.M., Canizares M., Perruccio A.V., Hogg-Johnson S., Gignac M.A. (2015). Benefits gained, benefits lost: Comparing baby boomers to other generations in a longitudinal cohort study of self-rated health. Milbank Quarterly, 93(1), 40-72.

Bhaskaran K., Douglas I., Forbes H., dos Santos-Silva I., Leon D.A., Smeeth L. (2014). Bodymass index and risk of 22 specific cancers: a population-based cohort study of 5.24 million UK adults. The Lancet, 384 (9945), 755-65. 
Bungum T., Satterwhite M., Jackson A. W., Morrow J., James R. (2003). The relationship of body mass index, medical costs, and job absenteeism. American Journal of Health Behavior, 27(4), 456-462.

Caman O.K., Calling S., Midlov P., Sundquist J., Sundquist K., Johansson S. (2013). Longitudinal age-and cohort trends in body mass index in Sweden - a 24-year follow-up study. BMC Public Health, 13, 893-893.

Castetbon K, Vernay M, Malon A, Salanave B, Deschamps V, Roudier C, Oleko A, Szego E, Hercberg S. (2009). Dietary intake, physical activity and nutritional status in adults: the French nutrition and health survey (ENNS, 2006-2007). British Journal of Nutrition, 102, 733-743.

Cawley J. (2004). The impact of obesity on wages. Journal of Human Resources, 39(2), 451 474. Retrieved from https://ideas.repec.Org/a/uwp/jhriss/v39y2004i2p451-474.html

Cheng F.W., Gao X., Mitchell D.C., Wood C., Still C.D., Rolston D., Jensen G.L. (2016). Body mass index and all-cause mortality among older adults. Obesity, 24 (10), 2232-2239. DOI: 10.1002/oby.21612

Cole T.J., Bellizzi M.C., Flegal K.M., Dietz W.H. (2000). Establishing a standard definition for child overweight and obesity worldwide: international survey. British Medical Journal, 320(7244), 1240-1243. DOI: 10.1136/bmj.320.7244.1240

Conley D., Glauber R. (2007). Gender, body mass, and socioeconomic status: new evidence from the PSID. Advances in health economics and health services research, 17, 253-275.

Danubio M.E., Miranda G., Vinciguerra M.G., Vecchi E., Rufo F. (2008). Comparison of selfreported and measured height and weight: implications for obesity research among young adults. Economics and human biology, 6(1), 181-90.

Dassonneville R. (2013). Questioning generational replacement. An age, period and cohort analysis of electoral volatility in the netherlands, 1971-2010. Electoral Studies, 32(1), 37-47. DOI: https://doi.org/10.1016/j.electstud.2012.09.004

Di Angelantonio E., Bhupathiraju S.N., Wormser D., Gao P., Kaptoge S., Berrington de Gonzalez A., ... Hu F. B. (2016). Body-mass index and all-cause mortality: individualparticipant-data meta-analysis of 239 prospective studies in four continents. The Lancet, 388(10046), 776-786.

Di Cesare M., Bentham J., Stevens G.A., Zhou B., Danaei G., Lu Y., ... NCD Risk Factor Collaboration (2016). Trends in adult body-mass index in 200 countries from 1975 to 2014: a pooled analysis of 1698 population-based measurement studies with 19.2 million participants. Lancet, 387(10026), 1377-1396.

Drøyvold W.B., Nilsen T.I.L., Kriiger O., Holmen T.L., Krokstad S., Midthjell K., Holmen J. (2006). Change in height, weight and body mass index: Longitudinal data from the HUNT study in Norway. International journal of obesity, 30, 935-939.

Ethan F., Christopher W, (2019). Analyzing Age-Period-Cohort Data: A Review and Critique. Annual Review of Sociology, 45(1), 467-492. DOI: https://doi.org/10.1146/annurev-soc073018-022616

Farhat T., Iannotti R.J., Simons-Morton B.G. (2010). Overweight, obesity, youth, and health-risk behaviors. American Journal of Preventive Medicine, 38(3), 258-267.

$\mathrm{Fu} \mathrm{W.} \mathrm{(2016).} \mathrm{Constrained} \mathrm{estimators} \mathrm{and} \mathrm{consistency} \mathrm{of} \mathrm{a} \mathrm{regression} \mathrm{model} \mathrm{on} \mathrm{a} \mathrm{lexis} \mathrm{diagram.}$ Journal of the American Statistical Association, 111(513), 180-199. DOI: 10.1080/01621459.2014.998761 
Godina E. (2011). Secular trends in some Russian populations. Anthropologischer Anzeiger, 68(4), 367-377.

Gordon-Larsen P., Wang H., Popkin B.M. (2014). Overweight dynamics in Chinese children and adults. Obesity Reviews, 15(S1), 37-48. DOI: 10.1111/obr.12121

Grigorieva M.A. (2015). Dynamics of Height, Weight and Body Mass Index of Real Generations of Russian People Aged 18-34 in 1810-1995. The Review of Economy, the Law and Sociology, 3, 198-202. (In Russ.).

Gu H., Shao S., Liu J., Fan Z., Chen Y., Ni J., ... Wang J. (2019). Age- and sex- associated impacts of body mass index on stroke type risk: A 27 -year prospective cohort study in a lowincome population in China. Frontiers in Neurology, 10, 1-9. DOI:

10.3389/fneur.2019.00456

Gurevich K.G., Poston W.S.C., Anders B., Ivkina M.A., Archangelskaya A., Jitnarin N., Starodubov V.I. (2017). Obesity prevalence and accuracy of BMI-defined obesity in Russian firefighters. Occupational Medicine, 67(1), 61-63. DOI: 10.1093/occmed/kqw136

Harrington M., Gibson S., Cottrell R.C. (2009). A review and meta-analysis of the effect of weight loss on all-cause mortality risk. Nutrition Research Reviews, 22(1), 93-108. DOI: 10.1017/S0954422409990035

Heo J., Beck A.N., Lin S.-F., Marcelli E., Lindsay S., Karl Finch B. (2018). Cohort-based income gradients in obesity among U.S. adults. American Journal of Human Biology, 30(2), e23084.

Holford T.R. (1983). The estimation of age, period and cohort effects for vital rates. Biometrics, 39(2), 311-324. DOI: 10.1016/0021-9681(85)90106-7

Hosseini M., Kelishadi R., Baikpour M., Ataei N., Qorbani M., Yousefifard M., ... Mohammad K. (2017). Age-period-cohort analysis of obesity and overweight in Iranian children and adolescents. International Journal of Endocrinology and Metabolism, 15(4), e13561. DOI: 10.5812/ijem. 13561

Hu Y., Malyutina S., Pikhart H., Peasey A., Holmes M.V., Hubacek J., ... Bobak M. (2017). The relationship between body mass index and 10-year trajectories of physical functioning in middle-aged and older Russians: Prospective results of the Russian HAPIEE study. The Journal of Nutrition, Health and Aging, 21(4), 381-388.

Huffman S.K., Rizov M. (2007). Determinants of obesity in transition economies: The case of Russia. Economics and Human Biology, 5(3), 379-391.

Ivanova E.I. (2014). Structural and societal factors in health of Russian population. RSUH/RGGU Bulletin "Philosophy. Sociology. Art Studies” Series, 4, 138-155. (In Russ.).

Jaacks L., Gordon-Larsen P., Mayer-Davis E., Adair L., Popkin B. (2013). Age, period and cohort effects on adult body mass index and overweight from 1991 to 2009 in China: the China Health and Nutrition Survey. International journal of epidemiology, 42(3), 828-837.

Jiang T., Gilthorpe M., Shiely F., Harrington J., Perry I., Kelleher C., Tu Y.-K. (2013). Ageperiod-cohort analysis for trends in body mass index in Ireland. BMC Public Health, 13, 889889.

Kalichman L., Livshits G., Kobyliansky E. (2006). Indices of body composition and chronic morbidity: A cross?sectional study of a rural population in central Russia. American Journal of Human Biology, 18(3), 350-358. 
Kant A.K., Graubard B.I., Kumanyika S.K. (2007). Trends in Black-White Differentials in Dietary Intakes of U.S. Adults, 1971-2002. American Journal of Preventive Medicine, 32(4), 264-272.e1. DOI:10.1016/j.amepre.2006.12.011

Kawachi A., Shimazu T., Budhathoki S., Sawada N., Yamaji T., Iwasaki M., ... Tsugane S. (2019). Association of BMI and height with the risk of endometrial cancer, overall and by histological subtype: a population-based prospective cohort study in Japan. European Journal of Cancer Prevention, 28(3), 196-202.

Kline B., Tobias J.L. (2008). The wages of BMI: Bayesian analysis of a skewed treatmentresponse model with nonparametric endogeneity. Journal of Applied Econometrics, 23(6), 767-793.

Kolarzyk E., Pac A., Shpakou A., Kleszczewska E., Klimackaya L., Laskiene S. (2012a). Nutrition of overweight and obese students. Central European Journal of Medicine, 7(5), 665-671. DOI: 10.2478/s11536-012-0028-6

Kolarzyk E., Shpakou A., Kleszczewska E., Klimackaya L., Laskiene S. (2012b). Nutritional status and food choices among first year medical students. Central European Journal of Medicine , 7, 396-408. DOI: 10.2478/s11536-011-0167-1

Kolosnitsyna M.G., Berdnikova A.N. (2009). Overweight: what are its costs and what could be done? Applied Econometrics, Russian Presidential Academy of National Economy and Public Administration (RANEPA), 15(3), 72-93. (In Russ.).

Kolosnitsyna M.G., Kulikova O.A. (2018). Overweight: socioeconomic factors and consequences. Demographic Review, 5(4), 92-124. (In Russ.).

Kozyreva P., Kosolapov M., Popkin B.M. (2016). Data Resource Profile: The Russia Longitudinal Monitoring Survey - Higher School of Economics (RLMS-HSE) Phase II: Monitoring the Economic and Health Situation in Russia, 1994-2013. International Journal of Epidemiology, 45(2), 395-401. DOI: https://10.1093/ije/dyv357

Krul A.J., Daanen H.A.M., Choi H. (2010). Self-reported and measured weight, height and body mass index (BMI) in Italy, the Netherlands and North America. The European Journal of Public Health, 21(4), 414-419. DOI:10.1093/eurpub/ckp228

Kuczmarski M. F., Kuczmarski R.J., Najjar M. (2001). Effects of age on validity of selfreported height, weight, and body mass index: Findings from the Third National Health and Nutrition Examination Survey, 1988-1994. Journal of the American Dietetic Association, 101(1), 28-34.

Kwok M.K., Tu Y.K., Kawachi I., Schooling C.M. (2017). Age-period-cohort analysis of trends in blood pressure and body mass index in children and adolescents in Hong Kong. Journal of epidemiology and community health, 71(12), 1161-1167.

Lainscak M., von Haehling S., Doehner W., Anker S. (2012). The obesity paradox in chronic disease: Facts and numbers. Journal of cachexia, sarcopenia and muscle, 3, 1-4. DOI: 10.1007/s13539-012-0059-5

Lambert A.A., Putcha N., Drummond M.B., Boriek A.M., Hanania N.A., Kim V., ... COPDGene Investigators (2017). Obesity is associated with increased morbidity in moderate to severe COPD. CHEST, 151(1), 68-77. DOI: 10.1016/j.chest.2016.08.1432

Ley C. J., Lees B., Stevenson J. C. (1992). Sex- and menopause-associated changes in body-fat distribution. The American Journal of Clinical Nutrition, 55(5), 950-954.

DOI:10.1093/ajen/55.5.950 
Maruyama S., Nakamura S. (2018). Why are women slimmer than men in developed countries? Economics and Human Biology, 30, 1-13.

Mason, K. O., Mason, W. M., Winsborough, H. H., \& Poole, W. K. (1973). Some methodological issues in cohort analysis of archival data. American Sociological Review, 38, 242-258.

Mironov B. (2003). Height and weight of Russians of the Stalin era. Demoscope Weekly, 129130. (In Russ.). URL: http://demoscope.ru/weekly/2003/0129/

Mironov B. (2012). The welfare of the population and revolutions in imperial Russia: XVIII early XX century. 2nd ed., Rev., Add. Moscow: Ves Mir Publishers. (In Russ.).

Mock C.N., Grossman D.C., Kaufman R.P., Mack C.D., Rivara F.P. (2002). The relationship between body weight and risk of death and serious injury in motor vehicle crashes. Accident Analysis and Prevention, 34(2), 221-228.

Must A., Dallal G., Dietz W. (1991a). Reference data for obesity: 85th and 95th percentiles of body mass index (wt/ht2) - a correction. The American Journal of Clinical Nutrition, 54(5), 773-773.

Must A., Dallal G., Dietz W. (1991b). Reference data for obesity: 85th and 95th percentiles of body mass index (wt/ht2) and triceps skinfold thickness. The American Journal of Clinical Nutrition, 53(4), 839-846.

Ng C., Corey P.N., Young T.K. (2012). Divergent body mass index trajectories between aboriginal and non-aboriginal canadians 1994-2009 4an exploration of age, period, and cohort effects. American Journal of Human Biology, 24 , 170-176.

Nooyens A.C., Visscher T.L., Verschuren W.M., Schuit A.J., Boshuizen H.C., van Mechelen W., Seidell J.C. (2009). Age, period and cohort effects on body weight and body mass index in adults: The Doetinchem Cohort Study. Public Health Nutrition, 12(6), 862-870. DOI: $10.1017 / \mathrm{S} 1368980008003091$

Ogden C.L., Carroll M.D., Lawman H.G., Fryar C.D., Kruszon-Moran D., Kit B.K., Flegal K.M. (2016). Trends in obesity prevalence among children and adolescents in the United States, 1988-1994 through 2013-2014. Journal of the American Medical Association, 315(21), 22922299. DOI: $10.1001 /$ jama.2016.6361

Pelzer B., te Grotenhuis M., Eisinga R., Schmidt-Catran A.W. (2015). The nonuniqueness property of the intrinsic estimator in APC models. Demography, 52, 315-327.

Rodriguez C., Freedland S.J., Deka A., Jacobs E.J., McCullough M.L., Patel A.V., ... Calle E.E. (2007). Body mass index, weight change, and risk of prostate cancer in the Cancer Prevention Study II Nutrition Cohort. Cancer Epidemiology and Prevention Biomarkers, 16(1), 63-69.

Rosenquist J., Lehrer S.F., O'Malley A., Zaslavsky A., Smoller J., Christakis N. (2014). Cohort of birth modifies the association between FTO genotype and BMI. Proceedings of the National Academy of Sciences, 112, 354-359.

Roth L.W., Allshouse A.A., Lesh J., Polotsky A.J., Santoro N. (2013). The correlation between self-reported and measured height, weight, and BMI in reproductive age women. Maturitas, $76,185-188$.

Russia Longitudinal Monitoring survey, RLMS-HSE (2020), conducted by the National Research University Higher School of Economics and ZAO "Demoscope" together with Carolina Population Center, University of North Carolina at Chapel Hill and the Institute of Sociology RAS. URL: https://www.hse.ru/rlms/spss (data downloaded on 17.03.2020). 
StataCorp. (2019a). Stata graphics reference manual: Release 16. College Station, TX: A Stata Press Publication.

StataCorp. (2019b). Stata base reference manual: Release 16. College Station, TX: A Stata Press Publication.

Su S.-Y., Lee W.-C. (2019). Age-period-cohort analysis with a constant-relative-variation constraint for an apportionment of period and cohort slopes. PLOS ONE, 14(12). DOI: 10.1371/journal.pone. 0226678

Twig G., Yaniv G., Levine H., Leiba A., Goldberger N., Derazne E., ... Kark J.D. (2016). Bodymass index in 2.3 million adolescents and cardiovascular death in adulthood. The New England Journal of Medicine, 374(25), 2430-2440.

Vandeschrick C. (2001). The Lexis diagram, a misnomer. Demographic Research, 4(3), 97124. DOI: 10.4054/DemRes.2001.4.3

Wahl S., Drong A., Lehne B., Loh M., Scott W.R., Kunze S., ... Chambers J.C. (2017). Epigenome-wide association study of body mass index, and the adverse outcomes of adiposity. Nature, 541(7635), 81-86. DOI: 10.1038/nature20784

Wang Y., Wang J. (2002). A comparison of international references for the assessment of child and adolescent overweight and obesity in different populations. European Journal of Clinical Nutrition, 56(10), 973-982. DOI: 10.1038/sj.ejcn.1601415

Wansink B., Cheney M., Chan N. (2003). Exploring comfort food preferences across age and gender1. Physiology \& Behavior, 79(4-5), 739-747. DOI:10.1016/s0031-9384(03)00203-8

Wardle J., Haase A.M., Steptoe A., Nillapun M., Jonwutiwes K., Bellisie F. (2004). Gender differences in food choice: The contribution of health beliefs and dieting. Annals of Behavioral Medicine, 27(2), 107-116. DOI:10.1207/s15324796abm2702_5

WHO (1995). Physical status: the use and interpretation of anthropometry (Tech. Rep.). WHO Technical Report Series No. 854. Geneva: World Health Organization.

Wilson R., Abbott J.H. (2018). Age, period and cohort effects on body mass index in New Zealand, 1997-2038. Australian and New Zealand Journal of Public Health, 42(4), 396-402.

Wooldridge J.M. (2010). Econometric Analysis of Gross Section and Panel Data. Cambridge, Massachusetts; London, England: The MIT Press.

Yang Y., Land K.C. (2013). Age-period-cohort analysis: New models, methods, and empirical applications. CRC Press.

Yang Y., Land, K.C. (2006). A mixed models approach to the age-period-cohort analysis of repeated cross-section surveys, with an application to data on trends in verbal test scores. Sociological Methodology, 36(1), 75-97. DOI: 10.1111/j.1467-9531.2006.00175.x

Yi S.-W., Ohrr H., Shin S.-A., Yi J.-J. (2015). Sex-age-specific association of body mass index with all-cause mortality among 12.8 million Korean adults: a prospective cohort study. International Journal of Epidemiology, 44(5), 1696-1705. DOI: 10.1093/ije/dyv138

Yu E., Ley S., E Manson J., Willett W., Satija A., B Hu F., Stokes A. (2017). Weight history and all-cause and cause-specific mortality in three prospective cohort studies. Annals of Internal Medicine, 166(9), 613-620.

Zagorsky J.L. (2005). Health and wealth: The late-20th century obesity epidemic in the U.S. Economics and Human Biology, 3(2), 296-313. 
Zhang L. (2015). How does age matter in the linkage between religious involvement and adult self-rated health? Advances in Applied Sociology, 5(4), 146-160.

DOI: $10.4236 /$ aasoci.2015.54014. 